\title{
NON-LINEAR RELATIONSHIPS BETWEEN HOUSE SIZE AND PRICE
}

\author{
Shih-Tao FENG ${ }^{1}$, Chien-Wen PENG ${ }^{1, *}$, Chung-Hsien YANG ${ }^{2}$, Pei-Wen CHEN ${ }^{1}$ \\ ${ }^{1}$ Department of Real Estate and Built Environment, National Taipei University, No. 151, University Rd., San Shia \\ District, 23741 New Taipei City, Taiwan \\ ${ }^{2}$ Department of Real Estate Management, National Pingtung University, No. 4-18, Minsheng Rd., \\ Pingtung City, 900391 Pingtung County, Taiwan
}

Received 21 April 2020; accepted 26 January 2021

\begin{abstract}
This study reexamines the relationship between house size and price by using the quantile regression model. Housing transactions data of the National Taipei University Special Zone in Taiwan are adopted, and the findings are as follows. First, the total price of a smaller housing unit will increase at a decreasing rate as its size increases. The decrease in marginal price might be due to the declining marginal utility of the property right. Secondly, the total price of a larger housing unit will increase at an increasing rate as its size increases. The size premium effect might be due to the influence of conspicuous consumption. Thirdly, housing with a lower square meter price is subject to greater price competitiveness in the market, and the price will increase at a decreasing rate as the size increases. Conversely, a housing unit with a higher square meter price will decrease at an increasing rate as its size increases. This might be due to the constraint imposed by the purchaser's housing affordability. These findings clarify the nonlinear relationships between housing size and price, and provide very useful information for decision making of the developers, home purchasers, real estate appraisers, and the governments.
\end{abstract}

Keywords: housing price, house size, size premium effect, size discount effect, non-linear relationship.

\section{Introduction}

When the buyers purchase a considerable number of commodities, the supplier is generally willing to provide a quantity discount. It is similar to the concept of switching from retail to wholesale or bulk sales. From the perspective of the supplier, it can achieve the purposes of promotion, reduce the frequency of transactions, and reduce transaction costs. From the perspective of the buyer, it is a type of reimbursement due to the decreasing marginal utility of consumption or opportunity cost of inventory. Unless there is conspicuous consumption or there are goods with economies of scale (such as land for development), there is rarely a norm that takes the form of a quantity premium effect.

As for housing, it has considerable features that are different from those of general commodities. The housing itself includes dual functions of consumption and investment. Moreover, there are features of fixed location, high heterogeneity, and durability. Once housing is completed, various features or attributes will become a set of packages. It is difficult to adjust feature combinations un- less housing undergoes major alterations or reconstruction in the future. Among many housing features, size is arguably the most important and least changeable feature. Therefore, the developer must be very cautious and precise during the product planning and design phase, otherwise possible losses might result due to errors in product positioning. Similarly, the household must fully consider the space requirement of family members before making a housing purchase. If the required space is miscalculated, the household must adjust their housing through selling and repurchasing new housing to make space adjustments. This will generate relatively high transaction costs.

According to the law of diminishing marginal utility, when the increase in house size exceeds the required optimal size of the purchaser, the willingness price to pay will decrease. As the total price will marginally increase with the increase of house size, there will be a quantity discount relationship between the size and price. This will be the main reason why the real estate appraiser will adjust the square meter price (hereinafter referred to as

*Corresponding author. E-mail: cwpeng@mail.ntpu.edu.tw

Copyright $\odot 2021$ The Author(s). Published by Vilnius Gediminas Technical University

This is an Open Access article distributed under the terms of the Creative Commons Attribution License (https://creativecommons.org/licenses/by/4.0/), which permits unrestricted use, distribution, and reproduction in any medium, provided the original author and source are credited. 
price-per-ping $)^{1}$ downwards when the value of the subject or comparable real estate exceeds a certain amount or the size exceeds a certain size in practice. However, if large housing has the feature of conspicuous consumption, the above adjustments will cause serious deviations. Moreover, different appraisers often have different ways of determining the standards for what constitutes a normal or optimal size, which in turn will raise doubts on excessively arbitrary or subjective price adjustments.

Previous studies on the relationship between real estate size and price are mostly related to the land market (Asabere \& Colwell, 1985; White, 1988; Colwell \& Scheu, 1989; Brownstone \& de Vany, 1991; Tabuchi, 1996; Thorsnes \& McMillen, 1998; Colwell \& Munneke, 1999; Thorsnes, 2000; Lin \& Evans 2000; Ecker \& Isakson, 2005; Clauretie \& Li, 2019; Ritter et al., 2020). In comparison to the land, the size differences between different housing alternatives are relatively small. However, the housing price exhibits a nonlinear relationship with the house size (Hamilton, 1976; Haurin, 1988; Turnbull et al., 2006; Leguizamon, 2010; Zahirovic-Herbert \& Chatterjee, 2011; Asabere \& Huffman, 2013; Simlai, 2014; Li et al., 2015; Lee \& Mori, $2016,2019)$. Since the empirical results of previous studies are quite strikingly different, it is necessary to clarify the relationship between the house size and price.

This research first establishes a theoretical model of house size and price (total price and price-per-ping), and then conducts empirical research by both OLS and quantile regression $(\mathrm{QR})$ to confirm the theoretical model. Comparing with previous studies, the main differences and contributions of this study are as follows. First, for the effective control of neighborhood factors that affect the housing price, this study selects the special district of National Taipei University (the NTPU Special Zone hereafter), an area with very similar neighborhood environment as the scope of the empirical analysis. Secondly, this study adopts the QR model for the empirical analysis. Compared with the ordinary least squares method, the QR model's parameter estimation can minimize the sum of the absolute values of all error terms and provide more robustness in relation to outliers. Thirdly, since the housing with a lower price-per-ping quantile is not necessarily in a lower quantile of the total price quantile, therefore, the housing price is divided into the price-per-ping and the total price. This paper gives a good overview about the upcoming challenges and methods for house size and price modelling and can be regarded as a reference for others with its conceptual intention. It also provides very useful information for decision making of the developers, home purchasers, real estate appraisers, and the governments.

This study that follows provides the literature review, the research methodology and research design, the empirical analysis, and the final section the conclusions and recommendations.

\footnotetext{
1 The "ping" is a local measurement of building size in Taiwan which following the usage of Japan. One ping equals to 3.3058 square meters. $1 \mathrm{USD}=28 \sim 30$ NTD.
}

\section{Literature review}

\subsection{Land size and price}

Most studies recognize that the land price will exhibit a nonlinear relationship with the increase in the size, however, the nonlinear relationships obtained by different empirical analyses are strikingly different. Some studies find that as the land size increases, the land price will exhibit a concave relationship with a decreasing marginal return. This is referred to as the quantity discount effect (Asabere \& Colwell, 1985; White, 1988; Colwell \& Scheu, 1989; Brownstone \& de Vany, 1991; Colwell \& Sirmans, 1993; Thorsnes \& McMillen, 1998). Conversely, some studies indicated that, as the size increases, the land price will exhibit a convex relationship with an increasing marginal return. This is referred to as the quantity premium effect (Tabuchi, 1996; Thorsnes, 2000; Lin \& Evans, 2000).

There are also studies that have found that the land price for different size intervals has exhibited both quantity premium effect and discount effect norms (Asabere \& Colwell, 1985; Colwell \& Munneke, 1999; Ecker \& Isakson, 2005; Clauretie \& Li, 2019). For example, Asabere and Colwell (1985) found that in comparison to the average size of vacant land, the price of a large-sized vacant land will be characterized by a discount effect, and a vacant land that is smaller than the average size will have a premium effect. Clauretie and $\operatorname{Li}$ (2019) found that the price paid per acre of land initially increases, and then decreases with parcel size. Convexity exists on the value-size curve up to approximately 8 acres, and the price paid per acre to be maximized at approximately 14 acres. However, Ritter et al. (2020) analyze a dataset of more than 80,000 agricultural land transactions in Germany. They concluded that the complex relationship between land price and plot size cannot be captured by a simple functional form since it is affected by several economic factors, such as economies of size, transaction costs, and financial constraints.

\subsection{House size and price}

Most studies have found that under the effect of the diminishing marginal utility law, larger-sized housing will give rise to a discount effect (Rosen, 1974; Turnbull et al., 2006; Asabere \& Huffman, 2013; Simlai, 2014). For example, Simlai (2014) argued that the total housing price would increase with the increase in its size, but that this tendency would decelerate as the size further increased. The marginal substitution rate of the house size and the willingness to pay would decrease. Asabere and Huffman (2013) summarized the relationships between the house size and price into seven effects, which were atypical, conspicuous consumption, financial capitalization, household affordability, management and maintenance costs, investment potential, and neighborhood search results.

Large housing with conspicuous consumption may have a quantity premium effect (Veblen, 1899; Lee et al., 2006; Leguizamon, 2010; Zahirovic-Herbert \& Chatterjee, 2011; Li et al., 2015; Hei et al., 2016; Lee \& Mori, 2016, 
2019). For example, Veblen (1899) argued that conspicuous goods could give the purchaser additional benefits beyond the goods themselves, such as revealing their wealth and social status. According to Veblen's theory, larger-sized housing may be easier to sell than ordinary housing and have a premium effect. Li et al. (2015) found that as the size of the housing becomes larger, the square feet price becomes higher. It can fully reveal the conspicuous nature of a person's wealth and social status for having larger-sized housing in Hong Kong. Similarly, Lee and Mori (2016) found that under the control of relevant demographic and economic features, conspicuous demand does have a significantly positive influence on the residential premium effect, and there are obvious regional and time differences. Conversely, smaller-sized housing may have a discount effect. Hui et al. (2016) argue that the amount of living space can reflect the economic situation of the purchaser. Since a larger house size results in a higher square feet price, the housing market in Hong Kong does not conform to the law of one price.

For smaller housing with fiscal capitalization, there will also be a quantity premium effect (Hamilton, 1976; Turnbull et al., 2006; Asabere \& Huffman, 2013). For example, Hamilton (1976) argued that local governments usually levy higher taxes on larger-sized housing and extend tax benefits to smaller-sized housing. This will cause larger-sized housing to become less attractive and more difficult to sell in the market, and will result in a discount effect for larger-sized housing. Smaller-sized housing, by contrast, can generate a premium effect. Asabere and Huffman (2013) also indicated that large-sized housing gave rise to a discount effect compared to average-sized housing, while small-sized housing had a premium effect. As for other atypical housing that is either too large or too small, there should be a quantity discount effect. Haurin (1988) argued that atypical housing, in comparison with mainstream products in the market, regardless of whether they were oversized or undersized, usually required a longer sales period. The transaction price would be lower than the market price.

Since the empirical results of previous studies are strikingly different, it is necessary to clarify the relationship between the housing price and house size. This study argues that the differences in the empirical results of previous studies may relate to the difference between the supply and demand structure or the model control variables in the actual situation. It is more appropriate to show that the empirical scope should be the same as the residential type and the local environmental homogeneity so that the relationship will not be biased due to incomplete control over the variables. Furthermore, Zietz et al. (2008) argued that the presupposition of using the OLS model is that the purchasers in the market are homogeneous. However, the market may have more than one set of prices because the purchasers are heterogeneous. In view of such concerns, the price can be divided into different quantiles using $Q R$ models.

\section{Research design}

\subsection{Hypotheses}

Most studies recognize in the past, most studies on house size and price only focused on the total price or price-perping. In this study, the total price of the housing and the price-per-ping have been integrated to establish a theoretical model of the relationship. First, the total price of the housing $(P)$ is the product of the price-per-ping $(p)$ and the size $(s), P=p s$. Although the total housing price is affected by size, it is also influenced by other housing hedonic vectors $(x)$. When determining the size, a developer that seeks to maximize profits will decide on the size in accordance with the housing price function of the market:

$$
P=f(s, x) \text {. }
$$

For ordinary housing, regardless of whether it is based on the decline in the marginal utility of the house size as in Simlai (2014) or the atypical residence which will generate a discount effect due to the extension of the sales period as in Haurin (1988) and Turnbull et al. (2006), the total price of the housing will increase with the increase in size. However, the marginal price will decrease as the size increase, which is expressed by:

$$
\begin{aligned}
& \frac{\partial P}{\partial s}>0 ; \\
& \frac{\partial}{\partial s}\left(\frac{\partial P}{\partial s}\right)=\frac{\partial^{2} P}{\partial s^{2}}<0 .
\end{aligned}
$$

Nevertheless, according to research by Lee et al. (2006), Li et al. (2015), and Lee and Mori (2016), if the high-priced housing is relatively scarce and has conspicuous consumption features, the marginal utility will not necessarily decrease with the increase in the size. However, despite the total housing price increasing with the increase in the size, as the marginal total price increases with the increase in the size, it will generate a quantity premium norm, which is:

$$
\frac{\partial}{\partial s}\left(\frac{\partial P}{\partial s}\right)=\frac{\partial^{2} P}{\partial s^{2}}>0 .
$$

Moreover, although Haurin (1988) believed that smaller-sized housing will also increase the length of the sales period due to its atypical nature, it will further generate a discount effect. However, this study claims that, within the Chinese ethnic society that views property rights with great importance, the price of a unit of smaller-sized housing (generally referred to as a suite) with a low total price may be higher than that of a unit of ordinary housing. As the suite reaches the minimum size threshold, it will record its highest price-per-ping. Then, the price-per-ping will decrease as the size increases. When the size exceeds the scope of a small suite, the price-per-ping will be the same as for other ordinary housing products. The reason may be that the quantity is relatively small and the exclusive price will be generated due to strong demand. In particular, in regions with a high degree of urbanization, high 
job opportunities, and relatively high housing prices, single people or newly-married couples can be the main appeal to such demand. In addition, from a property rights perspective, the suite is the smallest unit and a compromise is reached with a complete housing product portfolio. For a household that bought the suite by first renting, because the marginal utility of property rights conversion is relatively high and the total price is relatively low, the price will be higher than that of ordinary housing, but the suite's marginal utility will decrease as it increases in size.

Secondly, we derive the first derivative function of the price-per-ping $(p)$ and size $(s)$ based on the following:

$$
\begin{aligned}
& p=\frac{P}{s} \\
& \frac{\partial p}{\partial s}=\frac{\partial}{\partial s}\left(\frac{P}{s}\right)=\frac{\frac{\partial P}{\partial s} s-P}{s^{2}}=\frac{\frac{\partial P}{\partial s}-\frac{P}{s}}{s}=\frac{\frac{\partial P}{\partial s}-p}{s} .
\end{aligned}
$$

From this we derive:

$$
\begin{aligned}
& \frac{\partial p}{\partial s} \geq 0 \text { if } \frac{\partial P}{\partial s} \geq p ; \\
& \frac{\partial p}{\partial s}<0 \text { if } \frac{\partial P}{\partial s}<p .
\end{aligned}
$$

From Equation (6), it is found that when the marginal total price of the size is higher than the price-per-ping, the price-per-ping increases with the increase in size, and the two are characterized by a positive relationship with each other. On the contrary, from Equation (7), when the marginal total price of the size is lower than the price-perping, the price-per-ping will decrease with the increase in the size. The two will then be characterized by a negative relationship.

In the following, under the assumption that the marginal total price decreases with the increase in size in Equation (3), this study uses the second derivative function to further deduce whether the marginal price-perping will increase or decrease:

$$
\begin{aligned}
& \frac{\partial^{2} p}{\partial s^{2}}=\frac{\partial}{\partial s}\left(\frac{\partial s p}{\partial s}\right)=\frac{\partial}{\partial s}\left(p+s \frac{\partial p}{\partial s}\right)=\frac{\partial p}{\partial s}+\frac{\partial p}{\partial s}+s \frac{\partial^{2} p}{\partial s^{2}}= \\
& 2 \frac{\partial p}{\partial s}+s \frac{\partial^{2} p}{\partial s^{2}}<0 \\
& \frac{\partial^{2} p}{\partial s^{2}}<\left(\frac{-2}{s}\right) \frac{\partial p}{\partial s} \text { that is: } \\
& \frac{\partial^{2} p}{\partial s^{2}}<0 \text { if } \frac{\partial P}{\partial s} \geq p \\
& \frac{\partial^{2} p}{\partial s^{2}}<c \text { if } \frac{\partial P}{\partial s}<p(c>0) .
\end{aligned}
$$

From Equations (6) and (8), it is discovered that when the marginal total price of the size is higher than the price-per-ping, the price-per-ping will increase with the increase in the size, and the marginal price-per-ping will decline with the increase in the size. Conversely, from
Equations (7) and (9), when the marginal total price of the size is lower than the price-per-ping, the price-perping will decrease as the size increases. The trend of the marginal price-per-ping will be unclear, and the marginal price-per-ping may decline, but it may also increase. However, if the high-priced housing is considered to have a conspicuous consumption feature, the total marginal price will increase with the increase in size. The assumption is presented in Equation (4) as follows:

$$
\begin{aligned}
& \frac{\partial^{2} p}{\partial s^{2}}=\frac{\partial}{\partial s}\left(\frac{\partial s p}{\partial s}\right)=\frac{\partial}{\partial s}\left(p+s \frac{\partial p}{\partial s}\right)=\frac{\partial p}{\partial s}+\frac{\partial p}{\partial s}+s \frac{\partial^{2} p}{\partial s^{2}}= \\
& 2 \frac{\partial p}{\partial s}+s \frac{\partial^{2} p}{\partial s^{2}}>0 \\
& \frac{\partial^{2} p}{\partial s^{2}}>\left(\frac{-2}{s}\right) \frac{\partial p}{\partial s} \text { which is: } \\
& \frac{\partial^{2} p}{\partial s^{2}}>d \text { if } \frac{\partial P}{\partial s} \geq p(d<0) \\
& \frac{\partial^{2} p}{\partial s^{2}}>0 \text { if } \frac{\partial P}{\partial s}<p
\end{aligned}
$$

It is found from Equations (6) and (10) that when the marginal total price of the size is higher than the priceper-ping, the price-per-ping will then increase with the increase in the size. The marginal price-per-ping may increase with the increase in the size, but it may also decline as the size increases. On the contrary, from Equations (7) and (11), when the marginal total price of the size is lower than the price-per-ping, the price-per-ping will decrease with the increase in the size, and the marginal price-perping will increase with the increase in the size. When the size is a control variable, the total price has a positive relationship with the price-per-ping. The lower total price range means a lower price-per-ping range, whereas the higher total price range means a higher price-per-ping range. Therefore, this study can observe the high and low quantiles of the total price model and the price-per-ping model at the same time to test the features of small- and large-sized housing.

\subsection{Model and variables}

Lancaster (1966) developed a new theory of consumer demand, which arguing that what consumer are seeking to acquire is not goods themselves but the characteristics they contain. Rosen (1974) proposed that a class of differentiated products is completely described by a vector of objectively measured characteristics. Observed product prices and the specific amounts of characteristics associated with each good define a set of implicit or "hedonic" prices. This study uses the hedonic price theory to establish the hedonic price model as follows:

$$
\begin{aligned}
& P=\propto+X \beta+T \gamma+\varepsilon ; \\
& p=\mu+X v+T \xi+e,
\end{aligned}
$$

where: $P$ is the matrixes of total housing price; $p$ is the matrixes of price-per-ping; $\alpha$ and $\mu$ are the intercepts; $X$ is 
the characteristics matrixes of housing feature; $\beta$ and $v$ are the coefficient matrixes of housing feature; $T$ is dummy matrixes of transaction time; $\gamma$ and $\xi$ are the coefficient matrixes of transaction time; $\varepsilon$ and e are the error terms, respectively.

By referring to the model set of relevant studies in the second section and the transaction habits of the housing market in Taiwan. We classify the housing features into the housing units, building, and neighborhood environment features. The selected explainable variables include house size, land base size, located floor, number of rooms, number of living and dining rooms, number of bathrooms, height of floor, housing age, total floor, road width, public facility ratio, zone usage, building coverage ratio, distance from NTPU, distance from gateway, and distance from shops. For the definitions and expected signs of the variables, please refer to Table 1 .

By means of the OLS method and QR method, we can obtain the marginal willingness price of each housing feature for the housing price. This paper selects $0.1,0.25,0.5$, 0.75 and 0.9 quantiles for comparison. The five specific condition quantiles include the three quantiles $(0.25,0.5$, and 0.75 ) corresponding to the commonly used quartiles of the general QR and the $10 \%$ quantiles (0.1 and 0.9 ) of the left and right tails in the sample. We are then able to observe the features of the two tails in the sample distribution.

\subsection{Data}

This study utilizes the Actual Selling Price Registration Service Site from the Department of Land Administration, Ministry of Interior, and adopts real estate transaction data in the NTPU Special Zone (refer to Figure 1).

The influence of house size on price is strikingly different in the previous empirical studies. One possible reason might be the quality control of neighbor characteristics. For the effective control of neighborhood factors that affect the housing price, this study selects the NTPU Special Zone which located in Sanxia District of New Taipei City as the study area. The central and county governments started the planning of NTPU Special Zone in 1986 and opened the campus in 2000. To meet the foreseeable residential and commercial land demand that grew with the

Table 1. Variable definitions

\begin{tabular}{|c|c|c|}
\hline Variables & Definition & Exp. Sign \\
\hline Total price & Housing total price minus parking bay price & \\
\hline Price-per-ping & $\begin{array}{l}\text { Housing total price minus parking bay price, and then divided by the size minus } \\
\text { parking bay size }\end{array}$ & \\
\hline Size & Size recorded on the actual price registration site minus parking bay size & $+/-$ \\
\hline $\operatorname{Size}^{2}$ & As above & $+/-$ \\
\hline Land base size & Base size recorded on the building occupation permit & - \\
\hline $4^{\text {th }}$ floor & Housing unit situated on the 4 th floor? Yes $=1 / \mathrm{No}=0$ & - \\
\hline Top floor & Housing unit situated on the top floor: Yes $=1 / \mathrm{No}=0$ & $+/-$ \\
\hline No. of living and dining rooms & Number of living and dining rooms & + \\
\hline No. of rooms & Number of rooms & + \\
\hline No. of bathrooms & Number of bathrooms & + \\
\hline Height of floor & $\begin{array}{l}\text { Height from the interior floor surface to the above floor surface, in accordance with } \\
\text { the building occupation permit }\end{array}$ & + \\
\hline Housing age & The period from completion of the building & - \\
\hline Total floor & Total floors of the building & + \\
\hline Road width & Front entrance road width in various communities & + \\
\hline Public facility ratio & Public facility ratio of various communities & - \\
\hline Zone usage & Zone usage of the housing location: Residential $=1 /$ Commercial $=2$ & $+1-$ \\
\hline Building coverage ratio & Building coverage ratio recorded on the building occupation permit & - \\
\hline Distance from NTPU & Shortest walking distance from various communities to NTPU front or side entrances & - \\
\hline Distance from highway & The shortest distance from various communities to the highway & + \\
\hline Distance from gateway & Shortest walking distance from various communities to the gateway & - \\
\hline Distance from shops & Shortest walking distance from various communities to shops/stores & - \\
\hline Transaction period & $\begin{array}{l}\text { The base year is 2014. Five dummy variables are set, which are 2011, 2012, 2013, } \\
2015 \text { and } 2016\end{array}$ & $+/-$ \\
\hline
\end{tabular}

Note: We measure the walking distance between the housing unit and its nearby facilities based on the OSM (open street map) which provided by the local government. The only exception is the measurement of the distance between the specific housing unit to the highway which using the shortest distance on the map directly. 


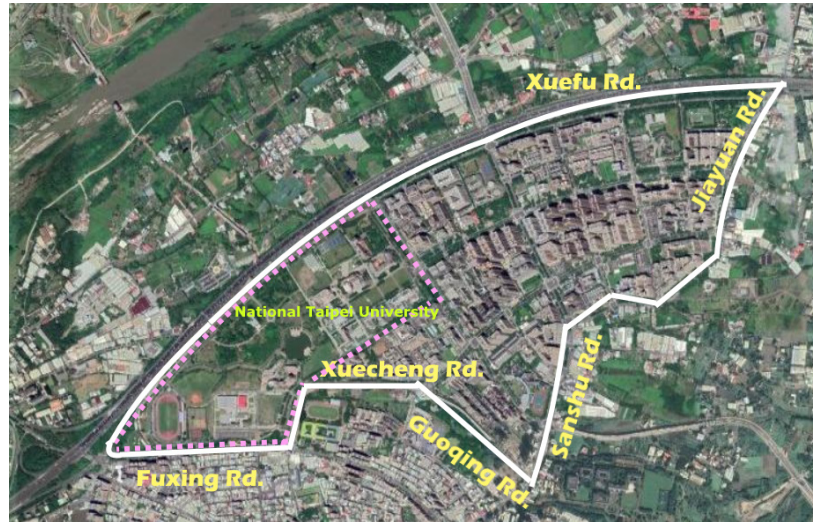

Figure 1. Background of National Taipei University Special Zone

development of the university, the government acquired all private land in the district through eminent domain. It is a newly developed area with very similar neighborhood environment, and the age of buildings are all below 15 years. With strict land-use control and urban design review regulations, the homogeneity of the NTPU Special Zone is relatively high.

The data's registered duration is from August 2012 to December 31,2016. There is a total of 5,620 observations.
By deducting the possible price influences, such as the ground floor, incomplete data, rapid sales, sales among family members and relatives, and commercial usage purposes, the final overall dataset consists of 5,021 observations. To ensure the consistent quality of the research data, apartments with no elevators, owner-occupied houses, storefronts and office buildings (accounting for about 2\% of the overall sample) are excluded in the data screening process. Other than that, to avoid the influence of parking bays, the parking bay size is deducted from the house size or housing price. The price-per-ping is calculated after the parking bay size and price is deducted from the house size and housing price. This is the basis for the calculation of the price-per-ping in this study.

\section{Empirical analysis}

Descriptive statistics, total price and price-per-ping model mark the results of empirical analysis.

\subsection{Descriptive statistics}

From the housing variable descriptive statistics in Table 2, the minimum price-per-ping is $141,710 \mathrm{NTD} /$ ping, the maximum price-per-ping is $377,810 \mathrm{NTD} /$ ping, the total

Table 2. Descriptive statistics of housing variables

\begin{tabular}{|c|c|c|c|c|c|c|}
\hline Variables & Type & Unit & Min & Max & Mean & Std. Dev. \\
\hline Total price & continuous & 10,000 NTD/ping & 14.17 & 37.78 & 25.79 & 3.71 \\
\hline Price-per-ping & continuous & 10,000 NTD & 165 & 5,000 & $1,189.02$ & 624.71 \\
\hline Size & continuous & ping & 8.79 & 174.45 & 43.01 & 20.07 \\
\hline Land base size & continuous & ping & 273.45 & $6,755.71$ & 2789.27 & 1466.94 \\
\hline $4^{\text {th }}$ floor & dummy & - & 0 & 1 & 0.07 & 0.25 \\
\hline Top floor & dummy & - & 0 & 1 & 0.08 & 0.27 \\
\hline No. of living and dining rooms & continuous & - & 0 & 8 & 1.75 & 0.55 \\
\hline No. of rooms & continuous & - & 1 & 8 & 2.86 & 0.98 \\
\hline No. of bathrooms & continuous & - & 1 & 8 & 1.84 & 0.75 \\
\hline Height of floor & continuous & $\mathrm{m}$ & 3 & 4.5 & 3.24 & 0.13 \\
\hline Housing age & continuous & year & 1 & 13 & 8.13 & 2.62 \\
\hline Total floor & continuous & floor & 5 & 28 & 17.32 & 4.98 \\
\hline Road width & continuous & $\mathrm{m}$ & 8 & 40 & 20.68 & 8.23 \\
\hline Public facility ratio & continuous & $\%$ & 12 & 40 & 28.59 & 2.72 \\
\hline Zone usage & dummy & - & 0 & 1 & 0.66 & 0.47 \\
\hline Building coverage ratio & continuous & $\%$ & 23.02 & 59.54 & 43.36 & 7.89 \\
\hline Distance from NTPU & continuous & $\mathrm{m}$ & 80 & 1500 & 744.55 & 365.53 \\
\hline Distance from highway & continuous & $\mathrm{m}$ & 40 & 1000 & 473.62 & 235.85 \\
\hline Distance from gateway & continuous & $\mathrm{m}$ & 300 & 1900 & 1206.19 & 364.77 \\
\hline Distance from shops & continuous & $\mathrm{m}$ & 58 & 1000 & 429.83 & 217.42 \\
\hline 2011 & dummy & - & 0 & 1 & 0 & 0.04 \\
\hline 2012 & dummy & - & 0 & 1 & 0.1 & 0.3 \\
\hline 2013 & dummy & - & 0 & 1 & 0.43 & 0.5 \\
\hline 2015 & dummy & - & 0 & 1 & 0.16 & 0.37 \\
\hline 2016 & dummy & - & 0 & 1 & 0.12 & 0.33 \\
\hline Sample size & & & & & & $n=5,021$ \\
\hline
\end{tabular}


price is $16,510,000 \mathrm{NTD}$, and the maximum total price is $50,001,000$ NTD. From the maximum and minimum of the total price and price-per-ping, it is apparent that the relatively high-priced housing does not necessarily have the highest price-per-ping in the area. Conversely, the relatively low-cost housing does not necessarily have the lowest price-per-ping in the area.

In terms of the distribution of the housing price-perping, the unitary skewness coefficient is 0.26 , and the kurtosis coefficient is 2.77 , which reflects an almost symmetric high-narrow peak distribution. It shows that the quality of the housing and price-per-ping is similar, where the housing price-per-ping in the NTPU Special Zone is characterized by a normal distribution. In terms of the distribution of the total price of the housing, the skewness coefficient of the total price is 1.43 , and the kurtosis coefficient is 5.73 , which is the distribution of the high-narrow peak of the right deviation. The average house size in the NTPU Special Zone is 42.96 pings. It is found that the house size transacted is mainly between 20 and 50 pings. For two observations, it is less than 20 pings or more than 70 pings, which accounts for $7.6 \%$ of the overall transaction sample.

\subsection{Results of total price model}

The explanatory power of the OLS regression model in Table 3 (adjusted $\mathrm{R}^{2}$ ) reached $94.94 \%$. Apart from the size and size squared VIF that exceeded 10 , there were no serious collinearity problems with the other variables. Size has a significant and positive influence on the total price at the $1 \%$ significance level and the square of size has a significant and negative influence at the $5 \%$ significance level. The total price will decrease with the size, and the marginal utility will decrease. As for the other explanatory variables, except for the top floor and the road width which are not significant and the sign for the building coverage ratio being different than expected, the signs of other variable are in line with expectations and have reached a significant level.

The explanatory power of the QR model is between 72\% and $84 \%$, and the model's explanatory power for the high quantile (total price) is higher than that for the low quantile (total price). Compared to the OLS regression model, the difference is that the influence of size in each quantile model has a positive effect at the $1 \%$ significance level. The value of the size coefficient declines from 25.47 to 24.30 as it shifts from the 0.1 quantile to the 0.25 quantile. Then, as the quantile increases to the 0.9 quantile, the coefficient's value is 28.82 . The 0.25 quantile has the smallest value. The square of the size has a significant negative influence in the case of the 0.1 quantile, and the other quantiles have a positive influence at the $1 \%$ significance level. Moreover, the influence increases with the increase in the quantile.

As the size coefficient rises from the 0.1 quantile to the 0.25 quantile, this causes the marginal total price to decline. It may be the case that housing with a total price at the 0.1 quantile has a higher marginal utility of property rights. The purchasers are willing to pay a higher price- per-ping, but the marginal utility of property rights will decrease as the size increases. This finding is consistent with the market behavior, especially in a country or region which highly value the homeownership.

To make more purchases in terms of size, the seller can only reduce the total price. The size coefficient value thus increases from the 0.25 quantile, reflecting the increase in the marginal total price of the size, and the purchasing price elasticity of the size is relatively small, which reflects the ability and willingness of people to purchase a larger size of housing. Under the assumption that the marginal cost does not change much, the property developer will have the incentive to provide housing units that are larger in size.

By using the least squares method, it is found that the land base size has a significant negative influence on the total price of the housing, which indicates that the greater the land base size, the lower the total housing price. A possible assumption is that the NTPU Special Zone is in the countryside of the Taipei Metropolitan Region. When the scale of the land base size is larger, the total price of housing is lower. If the developer plans its building with large-sized housing units in mind, it will put a lot of pressure on the sales team. The reason for this is that the housing purchaser who can purchase such a housing product also can purchase comfortable housing in the city center. Consequently, the opportunity cost of commuting will be relatively high. Faced with the commuting cost, for which the elasticity is greater than the income elasticity of the house size, the potential housing purchaser will tend to choose to live in the city center. Therefore, to avoid the pressure on future sales, the developer will tend to decide to build a number of small- and medium-sized housing units. However, from the QR, it is found that the land base size only has a significant positive influence on the total price at the 0.9 quantile. In other words, the finding indicates that the land base size only has a significant influence on a very small number of high-priced housing units. For low- to medium-priced housing, the influence of the land base size is not significant.

From the coefficient difference test between the different quantiles in Table 4 (the total price model), it is found that the size coefficient is significantly different between the 0.1 and 0.9 quantiles, and between the 0.5 and 0.9 quantiles. The difference in the size coefficient between the intermediate quantiles is not significant. From this it can be inferred that the total price per unit size of the high total price housing is higher than that of the low total price housing. At the 0.1 quantile, the size has a positive effect on the price, but the square of the size is negative. For the total price within the 4,951,000 NTD price band area, the size has a positive effect on the total price. However, as the size becomes larger, the total marginal price becomes lower. Conversely, the size is positively affected by the total price at the 0.9 quantile, and the square of the size is positively affected by the total price. This indicates that in between the high total price band, the total price will increase with the size and the total marginal price will increase as well. 


\begin{tabular}{|c|c|c|c|c|c|c|c|c|c|c|c|c|c|c|c|c|c|c|c|c|c|c|c|c|c|c|c|c|}
\hline \multirow{3}{*}{ 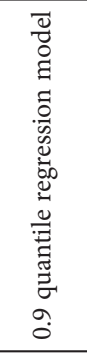 } & 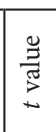 & I & $\begin{array}{l}\stackrel{\omega}{n} \\
\underset{+}{+}\end{array}$ & $\begin{array}{l}\infty \\
\infty \\
0 \\
0\end{array}$ & \begin{tabular}{l}
$:$ \\
$:$ \\
\hdashline
\end{tabular} & 7 & $=$ & $\begin{array}{l}\text { tu } \\
\text { i }\end{array}$ & 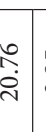 & $\begin{array}{l}\stackrel{2}{m} \\
\stackrel{n}{\xi}\end{array}$ & $\stackrel{\text { s. }}{-}$ & $\begin{array}{l}\stackrel{\infty}{i} \\
i \\
i\end{array}$ & $\underset{\leftarrow}{-}$ & $\stackrel{\infty}{\stackrel{\infty}{i}} \underset{i}{i}$ & L̊? & 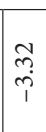 & $\stackrel{n}{\stackrel{n}{-}}$ & $\begin{array}{l}7 \\
\underset{1}{+} \\
+1\end{array}$ & in & $\begin{array}{l}\stackrel{\partial}{\vec{t}} \\
\vec{i}\end{array}$ & $\stackrel{\hat{m}}{i}$ & {$\left[\begin{array}{c}\stackrel{m}{0} \\
i n\end{array}\right]$} & तु & $\begin{array}{l}\text { त̂̀ } \\
\text { ị }\end{array}$ & $\underset{i}{+}$ & $\vec{m}$ & $\begin{array}{l}\stackrel{f}{2} \\
\dot{\varphi}\end{array}$ & \\
\hline & \multirow[b]{2}{*}{ 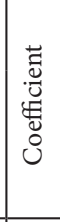 } & & 沜 & t. & 齐 & $\stackrel{x}{*}$ & 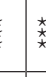 & & 奔 & 奔 & * & 䧇 & & $\stackrel{x}{\star}$ & & 委 & & $\begin{array}{l}\substack{x \\
x \\
x} \\
\end{array}$ & 亲 & & $\underset{\substack{x \\
\star}}{ }$ & $\underset{\substack{x \\
x}}{x}$ & $\underset{⿱}{x}$ & & & & $\underset{x}{x}$ & 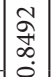 \\
\hline & & 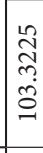 & 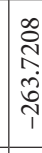 & $\mid \begin{array}{l}0 \\
0 \\
0 \\
0 \\
0 \\
1\end{array}$ & 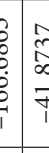 & 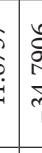 & & & $\begin{array}{c}H \\
\infty \\
\infty \\
\infty \\
\infty \\
\infty \\
\end{array}$ & 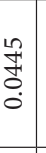 & $\begin{array}{l}\hat{\hat{o}} \\
\stackrel{0}{0} \\
\text {. }\end{array}$ & 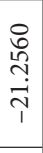 & 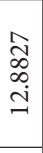 & 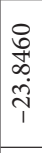 & $\stackrel{\text { in }}{\mathrm{m}}$ & 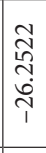 & $\begin{array}{l}\tilde{N} \\
\tilde{\delta} \\
\hat{N} \\
\hat{N}\end{array}$ & 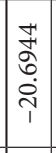 & $\begin{array}{l}2 \\
\text { 号 } \\
\text { N } \\
6\end{array}$ & 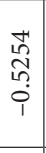 & $\begin{array}{l}0 \\
\stackrel{1}{1} \\
\stackrel{0}{0} \\
1\end{array}$ & 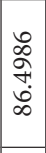 & 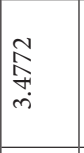 & $\begin{array}{l}0 \\
0 \\
0 \\
0 \\
i \\
1\end{array}$ & \begin{tabular}{|l}
$\overrightarrow{0}$ \\
0 \\
0 \\
$i$ \\
$i$
\end{tabular} & $\begin{array}{l}0 \\
\stackrel{0}{0} \\
0 \\
i \\
\end{array}$ & 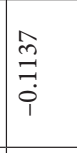 & \\
\hline \multirow{3}{*}{ 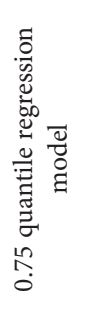 } & 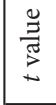 & $\stackrel{2}{-}$ & $\begin{array}{l}\exists \\
\text { I } \\
\text { i }\end{array}$ & $\frac{\substack{\infty \\
\tau}}{\tau}$ & 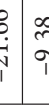 & $?$ & $\begin{array}{l}4 \\
i \\
i\end{array}$ & $\begin{array}{c}\text { ָे } \\
\infty \\
1 \\
1\end{array}$ & $\begin{array}{l}\stackrel{n}{a} \\
\text { i. }\end{array}$ & 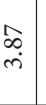 & 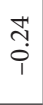 & $\begin{array}{l}\stackrel{n}{n} \\
i \\
i\end{array}$ & $\begin{array}{l}0 \\
\dot{0} \\
i\end{array}$ & $\underset{\substack{n \\
m \\
i}}{1}$ & $\tilde{g}$ & 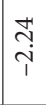 & 독 & 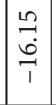 & 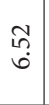 & 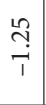 & $\hat{i}$ & $\dot{m}$ & 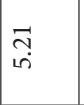 & ने & Oे & fr & $\hat{\bar{i}}$ & \\
\hline & \multirow{2}{*}{ 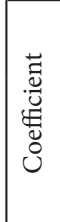 } & $\stackrel{\star}{\star}$ & 齐 & $\underset{\star}{\star}$ & 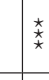 & $\stackrel{\substack{x \\
\star}}{*}$ & 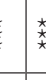 & & 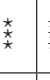 & 弃 & & $\stackrel{\star}{\star}$ & & 㭊 & * & 齐 & * & \begin{tabular}{|l|l}
$\substack{x \\
x}$ \\
\end{tabular} & 妾 & & $\stackrel{\star x}{\star}$ & 关 & 齐 & & & & $\underset{\substack{x \\
\star}}{*}$ & 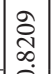 \\
\hline & & & $\begin{array}{l}\infty \\
\stackrel{\infty}{0} \\
\stackrel{1}{i} \\
\widetilde{N} \\
i \\
\end{array}$ & $\begin{array}{l}n \\
0 \\
\infty \\
0 \\
\infty \\
1 \\
1\end{array}$ & 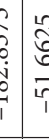 & 8 & & & 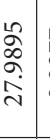 & $\begin{array}{l}\hat{\hat{O}} \\
\hat{o} \\
0\end{array}$ & $\begin{array}{l}\text { o } \\
\text { ठे } \\
\dot{i}\end{array}$ & $\stackrel{i}{i}$ & 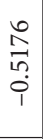 & 章 & $\begin{array}{l}\infty \\
\infty \\
\infty \\
\stackrel{n}{n}\end{array}$ & 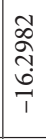 & $\begin{array}{l}0 \\
\text { o } \\
\text { d } \\
\text { i } \\
\text { in }\end{array}$ & 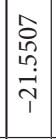 & 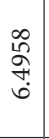 & $\begin{array}{l}\text { 索 } \\
\text { †े } \\
\vdots \\
i \\
\end{array}$ & 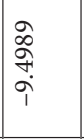 & 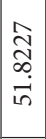 & 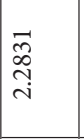 & $\mid \begin{array}{r}\hat{\theta} \\
\stackrel{0}{0}\end{array}$ & $\stackrel{0}{\stackrel{0}{a}}$ & \begin{tabular}{|}
0 \\
$\vdots$ \\
$\vdots$ \\
$\vdots$ \\
0 \\
0
\end{tabular} & $\begin{array}{c}n \\
\tilde{N} \\
\\
0 \\
1\end{array}$ & \\
\hline \multirow{3}{*}{ 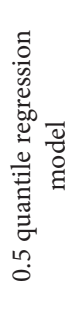 } & 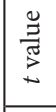 & $\stackrel{\sim}{\stackrel{n}{\rightarrow}}$ & $\begin{array}{l}\vec{i} \\
\vec{i}\end{array}$ & $\underset{\overbrace{}}{\tilde{\vartheta}}$ & $\begin{array}{l}0 \\
\vdots \\
\vdots \\
1\end{array}$ & & $\sigma_{0}$ & $\vec{i}$ & 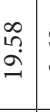 & $\begin{array}{l}\stackrel{0}{0} \\
\text { i }\end{array}$ & $\begin{array}{l}\tilde{6} \\
+ \\
+\end{array}$ & 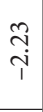 & 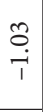 & $\begin{array}{l}\overrightarrow{0} \\
\dot{i}\end{array}$ & $\underset{i}{\stackrel{H}{i}}$ & $\begin{array}{c}\tilde{m} \\
\hat{i} \\
i\end{array}$ & $\stackrel{\infty}{\infty}$ & $\mid \begin{array}{c}\widetilde{\partial} \\
\stackrel{\vec{T}}{\mathrm{~T}}\end{array}$ & $\begin{array}{l}\hat{0} \\
\infty \\
\infty\end{array}$ & $\begin{array}{c}\stackrel{\infty}{0} \\
\dot{p} \\
i\end{array}$ & में & 家 & $\vec{i}$ & $\underset{\text { - }}{\text { Oे }}$ & 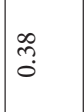 & 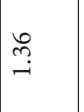 & $\underset{\substack{f\\
}}{=}$ & \\
\hline & \multirow{2}{*}{ 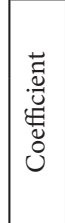 } & & 齐 & 龸 & 罙 & 龸 & $\frac{t}{*}$ & & t大 & 索 & & $\stackrel{\star}{\star}$ & & 沜 & $\stackrel{\star}{\star}$ & & * & 索 & 类 & 齐 & $\stackrel{\star}{\star}$ & * & 枽 & & & & 关 & $\begin{array}{l}\vec{\infty} \\
\stackrel{\infty}{\stackrel{0}{c}}\end{array}$ \\
\hline & & $\begin{array}{l}\hat{f} \\
\sigma \\
\dot{D} \\
\dot{D}\end{array}$ & $\begin{array}{l}m \\
\stackrel{2}{2} \\
2 \\
\\
7\end{array}$ & $\begin{array}{l}2 \\
2 \\
0 \\
2 \\
1\end{array}$ & 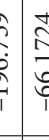 & $\hat{i}$ & 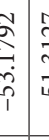 & & 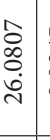 & $\begin{array}{l}\stackrel{2}{0} \\
\tilde{f} \\
0 \\
0\end{array}$ & $\begin{array}{l}F \\
\stackrel{+}{0} \\
\dot{i}\end{array}$ & 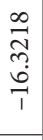 & 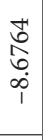 & 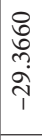 & $\begin{array}{l}\stackrel{0}{\alpha} \\
\hat{\sigma} \\
\end{array}$ & 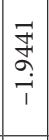 & $\begin{array}{l}\Re \\
\hat{\Omega} \\
\infty \\
\infty \\
i \infty\end{array}$ & 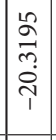 & 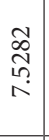 & 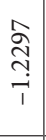 & $\begin{array}{l}\tilde{N} \\
\tilde{\infty} \\
\infty \\
i \\
1\end{array}$ & 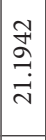 & సે & $\mid \begin{array}{l}\frac{1}{2} \\
0 \\
0 \\
0\end{array}$ & 会 & $\mid \begin{array}{l}\vec{\Delta} \\
\vec{\delta} \\
0\end{array}$ & $\begin{array}{c}\hat{\alpha} \\
\stackrel{7}{0} \\
i\end{array}$ & \\
\hline \multirow{3}{*}{ 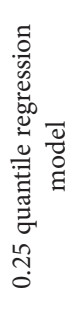 } & 总 & ه. & ஓे & 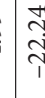 & 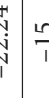 & $q a$ & & $\stackrel{m}{?}$ & $\begin{array}{l}\hat{\sigma} \\
\dot{m}\end{array}$ & $\begin{array}{l}\tilde{m} \\
\dot{m}\end{array}$ & $\begin{array}{l}\infty \\
\stackrel{\infty}{i}\end{array}$ & $\vec{m}$ & 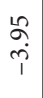 & 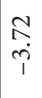 & $\stackrel{\infty}{\stackrel{\infty}{-}}$ & 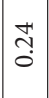 & $\cong$ & 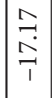 & $\mid \begin{array}{l}\infty \\
0 \\
\infty \\
\infty\end{array}$ & $\begin{array}{c}\overrightarrow{1} \\
\stackrel{1}{0} \\
\hat{1}\end{array}$ & 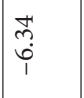 & भे & 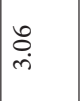 & 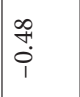 & 它 & $\mid \begin{array}{l}\infty \\
\vdots \\
i\end{array}$ & $\begin{array}{l}\hat{\infty} \\
\hat{p} \\
\hat{i}\end{array}$ & \\
\hline & \multirow{2}{*}{ 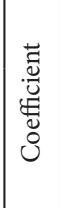 } & * & $\underset{\substack{x \\
x}}{*}$ & $\underset{\star}{\star}$ & $\underset{\star}{\star}$ & $\stackrel{\star x}{*}$ & $\begin{array}{l}\frac{x}{x} \\
\frac{x}{x}\end{array}$ & & 肴 & $\underset{\star}{\star}$ & & $\underset{⿱}{*}$ & $\underset{\star}{\star}$ & $\underset{\star}{x}$ & * & & * & $\underset{x}{*}$ & $\underset{\substack{x \\
\star}}{ }$ & $\underset{\star}{\star}$ & t & & $\underset{\star}{*}$ & & & & $\underset{\star}{\star}$ & 壳 \\
\hline & & $\begin{array}{l}\tilde{\aleph} \\
\hat{\lambda} \\
\hat{n} \\
\hat{n}\end{array}$ & 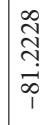 & 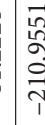 & 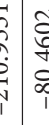 & & & $\begin{array}{l}\vec{w} \\
\vec{c} \\
\substack{0 \\
T}\end{array}$ & 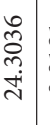 & $\begin{array}{l}\vec{a} \\
\vec{a} \\
\dot{0}\end{array}$ & $\begin{array}{l}\text { L్ } \\
\text { ô } \\
\dot{i}\end{array}$ & 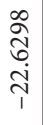 & 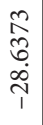 & $\begin{array}{l}\hat{\sigma} \\
\hat{c} \\
\infty \\
\infty \\
\rightarrow\end{array}$ & $\begin{array}{l}\hat{L} \\
\hat{O} \\
\infty \\
\infty\end{array}$ & 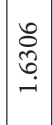 & 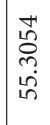 & 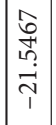 & $\begin{array}{l}n \\
\infty \\
0 \\
\sim \\
\infty \\
\infty\end{array}$ & $\mid \begin{array}{l}0 \\
0 \\
\infty \\
\stackrel{0}{0} \\
\rightarrow-1\end{array}$ & $\begin{array}{c}\vec{\infty} \\
\infty \\
0 \\
\infty \\
i\end{array}$ & $\begin{array}{l}\overrightarrow{\vec{D}} \\
\text { o } \\
\text { in }\end{array}$ & 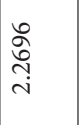 & 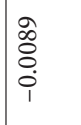 & 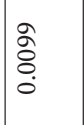 & $\mid \begin{array}{l}\infty \\
\stackrel{0}{0} \\
0 \\
i\end{array}$ & $\begin{array}{l}\text { for } \\
\stackrel{9}{0} \\
0\end{array}$ & \\
\hline \multirow{3}{*}{ 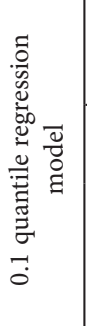 } & 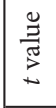 & $\stackrel{m}{m}$ & $\begin{array}{l}2 \\
\stackrel{2}{i} \\
\hat{i}\end{array}$ & $\mid \begin{array}{l}0 \\
0 \\
0 \\
1\end{array}$ & 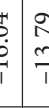 & $\vdots$ & id & $\begin{array}{l}7 \\
\text { in } \\
10\end{array}$ & $\begin{array}{l}\vec{a} \\
\dot{m} \\
\dot{m}\end{array}$ & $\begin{array}{c}\vec{A} \\
\dot{i}\end{array}$ & F! & $\begin{array}{l}+ \\
\dot{0} \\
\dot{P}\end{array}$ & $\begin{array}{l}\vec{D} \\
\dot{i} \\
i\end{array}$ & 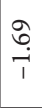 & $\stackrel{\overbrace{}}{=}$ & $\stackrel{n}{\rightarrow}$ & $\begin{array}{l}3 \\
0 \\
0\end{array}$ & 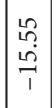 & $\begin{array}{l}\text { gे } \\
\text { in }\end{array}$ & $\underset{i}{\stackrel{m}{t}}$ & $\mid \begin{array}{l}\hat{A} \\
0 \\
i\end{array}$ & $\stackrel{0}{0}$ & ri & $\begin{array}{l}\hat{\beta} \\
i \\
i\end{array}$ & \&̊ & $\vec{i}$ & $\overrightarrow{\vec{p}}$ & \\
\hline & \multirow{2}{*}{ 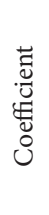 } & $\begin{array}{l}x \\
\star \\
\star\end{array}$ & & 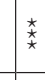 & $\underset{\star}{*}$ & 罙 & $\frac{t}{x}$ & & 齐 & $\underset{⿱}{x}$ & & $\underset{⿱}{\star}$ & 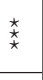 & * & & & & \begin{tabular}{|l|}
$\substack{x \\
x}$ \\
\end{tabular} & $\underset{\substack{x \\
\star}}{*}$ & & $\underset{*}{*}$ & & & 永 & & & $\underset{x}{x}$ & $|\underset{N}{\mathbb{N}}|$ \\
\hline & & 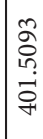 & $\begin{array}{l}\widetilde{1} \\
\infty \\
\omega \\
\infty \\
\infty \\
1\end{array}$ & 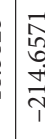 & 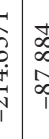 & 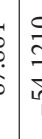 & 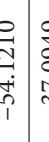 & 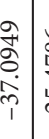 & 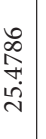 & 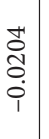 & $\begin{array}{l}0 \\
\stackrel{0}{0} \\
\stackrel{0}{\circ}\end{array}$ & 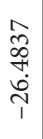 & 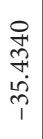 & $\begin{array}{l}\infty \\
0 \\
0 \\
0 \\
\hat{i} \\
\text {. }\end{array}$ & $\begin{array}{l}\text { 热 } \\
\text { م⿱ } \\
\text { b }\end{array}$ & $\begin{array}{c}\vec{n} \\
\hat{n} \\
\tilde{\sigma} \\
\alpha\end{array}$ & 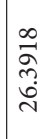 & 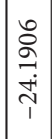 & \begin{tabular}{l}
$\infty$ \\
$o$ \\
\multirow{2}{*}{} \\
$\infty$ \\
$\infty$
\end{tabular} & 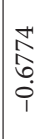 & $\begin{array}{l}0 \\
0 \\
\infty \\
0 \\
0 \\
1 \\
1\end{array}$ & 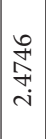 & | & 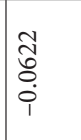 & 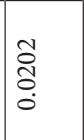 & $\begin{array}{l}0 \\
0 \\
0 \\
0 \\
0 \\
0 \\
1\end{array}$ & $\begin{array}{l}0 \\
0 \\
0 \\
0 \\
0 \\
i\end{array}$ & \\
\hline \multirow{4}{*}{ 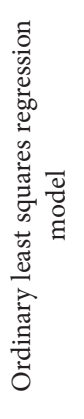 } & 岁 & & $\stackrel{\overbrace{}}{\text { }}$ & f & $f$ & & 6 & $\stackrel{\infty}{\rightarrow}$ & $\begin{array}{l}\text { d. } \\
\text {. } \\
\end{array}$ & $\begin{array}{l}\widetilde{\sigma} \\
\stackrel{\infty}{-}\end{array}$ & $\underset{m}{\stackrel{m}{m}}$ & 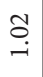 & $\underset{+}{\stackrel{H}{~}}$ & గ̂t & 돌 & $\begin{array}{l}\infty \\
\stackrel{\infty}{+} \\
\dot{m}\end{array}$ & $\stackrel{\substack{\infty \\
-\rightarrow}}{-}$ & $\overrightarrow{\tilde{i}}$ & \begin{tabular}{|c|}
$\overrightarrow{0}$ \\
$\dot{m}$
\end{tabular} & $\underset{\substack{\infty \\
-\infty}}{ }$ & 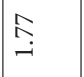 & 6 & $\dot{m}$ & 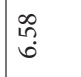 & î̀ & $\mid \begin{array}{c}\infty \\
\infty \\
\dot{0}\end{array}$ & $\begin{array}{l}\stackrel{\circ}{0} \\
\text { in }\end{array}$ & \\
\hline & 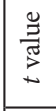 & $\vec{i}$ & $\begin{array}{c}\infty \\
\infty \\
i \\
i\end{array}$ & $\begin{array}{l}\partial \\
\infty \\
c\end{array}$ & 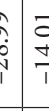 & 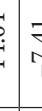 & $\underset{1}{F}$ & $\begin{array}{l}\text { tu } \\
0 \\
0 \\
1\end{array}$ & $\begin{array}{l}\text { Lे } \\
\text { d. } \\
i n n\end{array}$ & $\underset{i}{\vec{i}}$ & 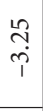 & $\begin{array}{c}\infty \\
\infty \\
\dot{p} \\
\dot{p}\end{array}$ & $\underset{t}{\rightleftarrows}$ & $\begin{array}{l}\infty \\
\stackrel{\infty}{i} \\
m_{1}^{2}\end{array}$ & $\stackrel{5}{9}$ & $\begin{array}{l}\vec{b} \\
i\end{array}$ & $\underset{+}{\stackrel{\infty}{+}} \underset{+}{+}$ & $\begin{array}{l}\hat{N} \\
\tilde{i} \\
i\end{array}$ & $\begin{array}{l}\stackrel{\partial}{\mathrm{j}} \\
\mathrm{J}\end{array}$ & 象 & 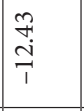 & | & Fi & $\underset{f}{\stackrel{f}{+}}$ & 4 & 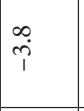 & 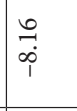 & \\
\hline & \multirow{2}{*}{ 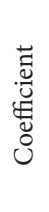 } & $\stackrel{\star}{\star}$ & $\underset{⿱}{*}$ & $\underset{x}{*}$ & $\underset{\star}{\star}$ & $\stackrel{\star}{*}$ & & & 齐 & $\stackrel{\star}{\star}$ & 索 & 䧇 & & 版 & 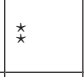 & 索 & 妾 & 永 & 齐 & & $\frac{x}{x}$ & 紊 & 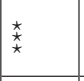 & 永 & $\stackrel{x}{*}$ & 亲 & 素 & 0 \\
\hline & & 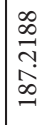 & 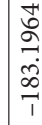 & 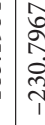 & 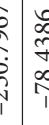 & & & & 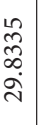 & 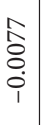 & $\begin{array}{l}\infty \\
\stackrel{0}{o} \\
\stackrel{0}{i}\end{array}$ & $\begin{array}{l}\vec{f} \\
\vec{n} \\
\dot{0} \\
\dot{p} \\
i\end{array}$ & $\begin{array}{l}m \\
3 \\
i \\
i \\
0 \\
1 \\
1\end{array}$ & 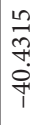 & $\begin{array}{l}\vec{D} \\
\infty \\
\vec{\sigma}\end{array}$ & $\begin{array}{l}\stackrel{\Im}{Z} \\
\grave{I} \\
\end{array}$ & $\begin{array}{l}n \\
\frac{n}{2} \\
\overrightarrow{9} \\
\alpha \\
\alpha\end{array}$ & 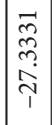 & $\begin{array}{l}\vec{F} \\
0 \\
0 \\
0\end{array}$ & $\begin{array}{l}\hat{\partial} \\
\stackrel{\partial}{0} \\
\overline{0}\end{array}$ & 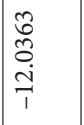 & $\begin{array}{l}\Xi \\
\vec{m} \\
\dot{\infty} \\
0\end{array}$ & $\underset{:}{\stackrel{0}{=}}$ & 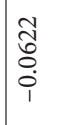 & 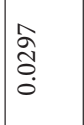 & $\begin{array}{l}7 \\
0 \\
0 \\
0 \\
0\end{array}$ & 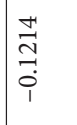 & \\
\hline $\begin{array}{l}\frac{\vec{v}}{0} \\
\frac{0}{\Sigma}\end{array}$ & 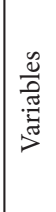 & 蒄 & & & & & 点 & c) & & के & 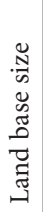 & 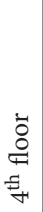 & & 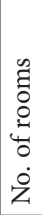 & 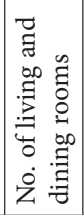 & 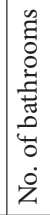 & 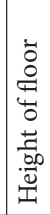 & 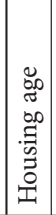 & 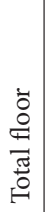 & 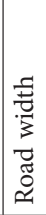 & 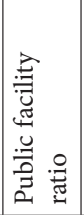 & 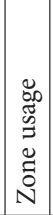 & 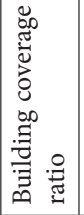 & 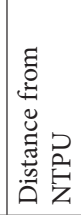 & 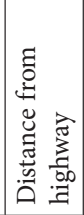 & 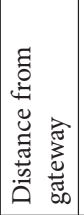 & 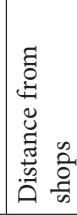 & 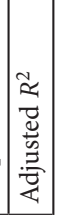 \\
\hline
\end{tabular}


Table 4. Housing total price model - coefficient difference test results for different quantiles

\begin{tabular}{|c|c|c|c|c|c|c|c|c|c|c|}
\hline \multirow{2}{*}{$\begin{array}{c}\text { Model } \\
\text { Variables }\end{array}$} & \multicolumn{2}{|c|}{$\begin{array}{l}0.1 \text { and } 0.25 \\
\text { quantiles }\end{array}$} & \multicolumn{2}{|c|}{$\begin{array}{l}0.1 \text { and } 0.5 \\
\text { quantiles }\end{array}$} & \multicolumn{2}{|c|}{$\begin{array}{c}0.5 \text { and } 0.9 \\
\text { quantiles }\end{array}$} & \multicolumn{2}{|c|}{$\begin{array}{c}0.75 \text { and } 0.9 \\
\text { quantiles }\end{array}$} & \multicolumn{2}{|c|}{$\begin{array}{l}0.1 \text { and } 0.9 \\
\text { quantiles }\end{array}$} \\
\hline & Coefficient & $t$ & Coefficient & $t$ & Coefficient & $t$ & Coefficient & $t$ & Coefficient & $t$ \\
\hline 2011 & $62.6405^{\star * *}$ & 2.35 & $160.8091^{\star * *}$ & 8.53 & $84.3295^{\star \star \star}$ & 4.54 & $31.6120^{*}$ & 1.61 & $245.1386^{\star * *}$ & 5.46 \\
\hline 2012 & -3.7020 & -0.34 & -17.8980 & -1.33 & $-30.0727^{\star * *}$ & -3.22 & $-16.171^{\star}$ & -1.56 & $-47.9707^{\star * *}$ & -3.88 \\
\hline 2013 & -7.4238 & -1.25 & $-21.7116^{\star * *}$ & -3.65 & $-24.2987^{\star * *}$ & -3.5 & $-9.7888^{\star}$ & -1.72 & $-46.0103^{\star * *}$ & -5.88 \\
\hline 2015 & 3.7153 & 0.49 & -0.9419 & -0.11 & $-18.3886^{\star \star}$ & -2.82 & -7.4561 & -0.99 & $-19.3305^{\star}$ & -2.53 \\
\hline 2016 & $11.1182^{\star}$ & 1.4 & $14.2179^{\star}$ & 1.87 & -1.1119 & -0.12 & -5.0505 & -0.54 & 13.1059 & 1.15 \\
\hline Size & 1.1750 & 1.17 & -0.6021 & -0.38 & $-2.7477^{\star}$ & -2.49 & -0.8389 & -0.96 & $-3.3498^{\star *}$ & -2.44 \\
\hline Size $^{2}$ & $-0.0395^{\star * *}$ & -4.88 & $-0.0549^{* * *}$ & -4.23 & -0.010 & -0.86 & -0.0068 & -0.79 & $-0.0649^{\star * *}$ & -4.56 \\
\hline Land base size & 0.0041 & 0.94 & 0.0057 & 1.73 & $-0.0120^{\star * *}$ & -3.28 & $-0.0087^{\star *}$ & -3.13 & -0.0063 & -1.11 \\
\hline $4^{\text {th }}$ floor & -3.8538 & -0.95 & -10.1619 & -1.03 & 4.9342 & 0.62 & 1.2560 & 0.14 & -5.2277 & -0.42 \\
\hline Top floor & -6.7967 & -0.62 & $-26.7576^{\star *}$ & -2.16 & $-21.5591^{\star *}$ & -2.19 & $-13.4003^{*}$ & -2.16 & $-48.3167^{\star * *}$ & -3.06 \\
\hline No. of rooms & 8.6441 & 1.28 & $19.7062^{\star \star}$ & 2.12 & -5.5199 & -0.61 & -9.7313 & -1.27 & 14.1862 & 1.21 \\
\hline No. of living and dining rooms & -1.2528 & -0.34 & -1.1829 & -0.23 & 4.4376 & 0.79 & 3.7833 & 0.81 & 3.2547 & 0.46 \\
\hline No. of bathrooms & 7.7245 & 1.44 & 11.2992 & 1.56 & $24.3081^{\star * *}$ & 3.06 & 9.9540 & 1.69 & $35.6073^{\star * *}$ & 3.09 \\
\hline Height of floor & -28.9136 & -0.65 & -31.6875 & -0.63 & -19.5529 & -0.4 & -25.5842 & -0.63 & -51.2404 & -0.9 \\
\hline Housing age & $-2.6439^{*}$ & -1.55 & $-3.8710^{\star \star}$ & -2.31 & 0.3749 & 0.21 & -0.8562 & -0.62 & $-3.4961^{\star}$ & -1.96 \\
\hline Total floor & -0.0086 & -0.01 & 0.7216 & 0.64 & 1.2828 & 1.38 & 0.2504 & 0.23 & 2.0044 & 1.43 \\
\hline Road width & $0.7089^{\star}$ & 1.94 & 0.5523 & 1.59 & -0.7043 & -1.74 & 0.0280 & 0.06 & -0.1520 & -0.34 \\
\hline Public facility ratio & -1.9979 & -1.45 & -2.5328 & -1.7 & 2.3198 & 1.66 & 0.6742 & 0.49 & -0.213 & -0.14 \\
\hline Zone usage & -3.5068 & -0.26 & -18.7195 & -1.37 & $-65.3045^{\star * \star}$ & -4.64 & $-34.6760^{\star *}$ & -2.36 & $-84.024^{\star * *}$ & -4.09 \\
\hline Building coverage ratio & -0.6561 & -0.79 & -0.1194 & -0.12 & $-1.7443^{* *}$ & -2.12 & $-1.194029^{* *}$ & -2.36 & -1.8637 & -1.34 \\
\hline Distance from NTPU & $-0.0533^{\star * *}$ & -2.51 & $-0.0797^{\star \star \star}$ & -4.34 & 0.0236 & 1.04 & 0.0133 & 0.57 & $-0.0560^{\star}$ & -2.38 \\
\hline Distance from highway & 0.0104 & 0.68 & 0.0146 & 0.55 & 0.0088 & 0.53 & 0.0047 & 0.2 & 0.0234 & 0.86 \\
\hline Distance from gateway & $-0.0443^{* *}$ & -1.94 & $-0.0797^{* * *}$ & -3.85 & 0.0277 & 1.2 & 0.0126 & 0.58 & $-0.0520^{*}$ & -2.55 \\
\hline Distance from shops & 0.0197 & 1.2 & $0.0547^{\star *}$ & 2.17 & -0.0260 & -1.44 & -0.0215 & -1.17 & 0.0287 & 1.02 \\
\hline
\end{tabular}

Note: ${ }^{*}{ }^{* *}$ and ${ }^{* * *}$ denote statistical significance at the $10 \%, 5 \%$, and $1 \%$ significance levels.

\subsection{Results of price-per-ping model}

As far as the OLS model is concerned, the size has no significant influence on the price-per-ping, and the result in Table 5 (the ping price model) is obviously different from that for the total price. As for the other variables, most of the variables that are concerned with distance do not reach a significant level in comparison to the result for the total price model. The sign for the top floor is also different from that expected. From the perspective of the QR model in Table 5 (the ping price model), the significance of size on the price-per-ping is only noticeable at the 0.1 and 0.9 quantiles, and shows that there is significance in terms of both positive and negative influences, respectively. Size has no significant effect on the price-per-ping in the cases of the intermediate quantiles.

At the 0.1 quantile, the size has a positive effect on the price-per-ping at the $1 \%$ significance level. This means that the price-per-ping will increase with the increase in the size and the marginal price-per-ping will decrease. This may be because the housing with a low price-per-ping imposes no obvious burden on the total price per unit size, and it is still within the affordable range of the purchaser. Therefore, for purchases of low-priced housing, the developer can increase the price-per-ping to maximize the return on investment. Moreover, at the 0.9 quantile, the size has a negative influence on the price-per-ping at the 5\% significance level. This means that the price-per-ping will decrease with the increase in the size and the marginal price-per-ping will increase. This may be because, when the price-per-ping is high, the increase in the total price will easily exceed the purchaser's burden limit. Therefore, when the developer is situated in locations where the price-per-ping range is higher than in the regional market, the size of the building unit should not be too large. The housing unit that is too large will have a negative influence on the price-per-ping.

From the coefficient difference test between the different quantiles in Table 6 (the ping price model), it is found that the size coefficient is significantly different between the 0.1 and 0.25 quantiles, between the 0.1 and 0.5 quantiles and between the 0.75 and 0.9 quantiles. From this it can be inferred that there is significant difference between the relatively low ping price and the high ping price. This also indicates that in the low ping price band, the ping price will increase with the size. 


\begin{tabular}{|c|c|c|c|c|c|c|c|c|c|c|c|c|c|c|c|c|c|c|c|c|c|c|c|c|c|c|c|c|}
\hline \multirow{3}{*}{ 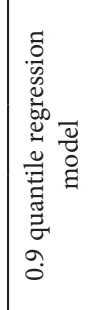 } & $\frac{\pi}{\pi}$ & $\stackrel{D}{\infty}_{\infty}$ & 走 & \begin{tabular}{l}
2 \\
2 \\
0 \\
\hdashline
\end{tabular} & 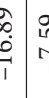 & 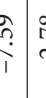 & $\begin{array}{l}\infty \\
\stackrel{1}{1} \\
i\end{array}$ & $\stackrel{\vec{m}}{\underset{+}{+}}$ & 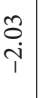 & $\underset{\mathrm{i}}{\mathrm{i}}$ & $\stackrel{\tilde{\sigma}}{\rightarrow}$ & $\stackrel{\substack{n \\
\rightarrow}}{\rightarrow}$ & $\stackrel{\overbrace{}}{\rightarrow}$ & $\begin{array}{l}\infty \\
\stackrel{\infty}{i} \\
i\end{array}$ & $\begin{array}{l}\infty \\
\hat{1} \\
i \\
i\end{array}$ & 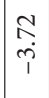 & $\begin{array}{c}\stackrel{n}{0} \\
0\end{array}$ & $\underset{+}{\stackrel{9}{+}}$ & $\ddot{i n}$ & $\begin{array}{l}0 \\
⿱ 亠 䒑 \\
i \\
1\end{array}$ & $\overrightarrow{\vec{p}}$ & 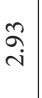 & & $\begin{array}{l}\stackrel{\infty}{0} \\
i\end{array}$ & 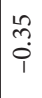 & 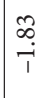 & $\begin{array}{c}\mathfrak{S} \\
\dot{i}\end{array}$ & \\
\hline & $\vec{\Xi}$ & $\underset{x}{*}$ & $\underset{\star}{\star}$ & $\stackrel{\star}{\star}$ & 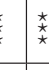 & 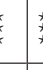 & 㐬 & 泫 & * & $\stackrel{\star}{\star}$ & * & & * & $\underset{x}{\star x}$ & & 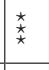 & & $\underset{\star}{\star}$ & $\underset{\underset{\star}{\star}}{\stackrel{\star}{*}}$ & & $\underset{⿱}{\star}$ & 齐 & $\underset{⿱}{x}$ & $\stackrel{\star}{*}$ & & * & $\underset{x}{*}$ & \multirow{2}{*}{ กֶ. } \\
\hline & $\begin{array}{l}\overrightarrow{\breve{y}} \\
\dot{\mathscr{\nu}} \\
\dot{0}\end{array}$ & $\begin{array}{l}\hat{\alpha} \\
\hat{\alpha} \\
\dot{y} \\
\dot{y}\end{array}$ & $\frac{8}{13}$ & 辛 & 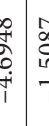 & 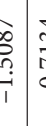 & 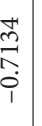 & 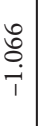 & 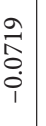 & $\begin{array}{l}\hat{o} \\
\stackrel{0}{\circ} \\
\circ\end{array}$ & 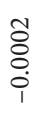 & $\begin{array}{l}\text { 令 } \\
\text { ñ. } \\
\text { ? }\end{array}$ & 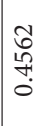 & $\begin{array}{l}0 \\
\overrightarrow{0} \\
0 \\
0 \\
1\end{array}$ & 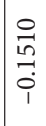 & 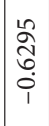 & $\mid \begin{array}{c}0 \\
0 \\
0 \\
0 \\
0\end{array}$ & 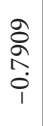 & 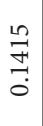 & $\mid \begin{array}{l}0 \\
\vdots \\
0 \\
\dot{1} \\
1\end{array}$ & $\begin{array}{l}\text { ثें } \\
\text { ते } \\
\text { i }\end{array}$ & 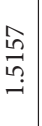 & $\begin{array}{l}\overrightarrow{\tilde{s}} \\
\stackrel{8}{0}\end{array}$ & $\begin{array}{l}0 \\
\overrightarrow{8} \\
0 \\
\end{array}$ & $\begin{array}{l}\hat{0} \\
\vdots \\
0 \\
i\end{array}$ & 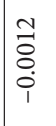 & 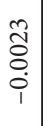 & \\
\hline \multirow{3}{*}{ 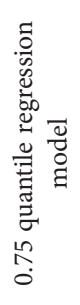 } & 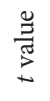 & $\begin{array}{l}\overrightarrow{0} \\
\stackrel{\square}{-}\end{array}$ & $\frac{12}{7}$ & 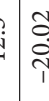 & 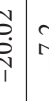 & $\begin{array}{c}c \\
1 \\
1\end{array}$ & ô & 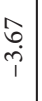 & 足 & $\underset{i}{\stackrel{+}{i}}$ & $\begin{array}{l}\text { fि } \\
\text { î }\end{array}$ & $\begin{array}{l}\underset{\infty}{\infty} \\
\stackrel{T}{i}\end{array}$ & $\stackrel{m}{0}$ & 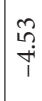 & $\hat{i}$ & $\overrightarrow{\vec{a}}$ & 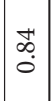 & 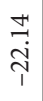 & के & 苗 & 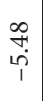 & 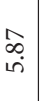 & $\stackrel{\infty}{\stackrel{\infty}{\rightarrow}}$ & $\begin{array}{l}\infty \\
i \\
i\end{array}$ & $\underset{\text { Ju }}{\text { OH }}$ & $\stackrel{H}{\sharp}$ & 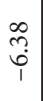 & \\
\hline & \multirow{2}{*}{ 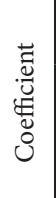 } & 齐 & $\underset{⿱ 亠 乂}{*}$ & $\underset{⿱ 亠 乂}{*}$ & $=\sqrt{x}$ & & $\frac{\sqrt{x}}{x}$ & 齐 & & 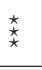 & & 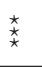 & & 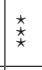 & 齐 & * & & $\underset{⿱}{*}$ & $\stackrel{\star \star ⿱ 乛 龰 ⿱ 十}{\star}$ & & $\underset{⿱ 亠 乂}{*}$ & 永 & * & $\stackrel{\star}{\star}$ & & & \multirow{2}{*}{\multicolumn{2}{|c|}{ 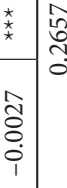 }} \\
\hline & & 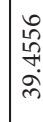 & 尽 & F & 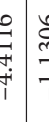 & 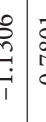 & 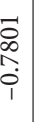 & 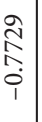 & 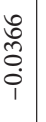 & 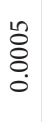 & 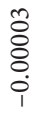 & $\begin{array}{l}\text { N } \\
\text { ît } \\
\text { in } \\
\text { int }\end{array}$ & 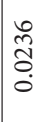 & 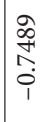 & $\begin{array}{l}\infty \\
\infty \\
\infty \\
i \\
i\end{array}$ & 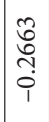 & $\mid \begin{array}{l}\infty \\
\stackrel{2}{\hat{\delta}} \\
\hat{0}\end{array}$ & 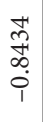 & $\begin{array}{l}\stackrel{H}{H} \\
\stackrel{6}{0} \\
0\end{array}$ & $\begin{array}{l}0 \\
0 \\
0 \\
0 \\
1\end{array}$ & $\begin{array}{l}\beth \\
\underset{1}{I} \\
\stackrel{0}{1}\end{array}$ & 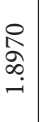 & 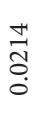 & $\begin{array}{l}\text { के } \\
\text { ¿े } \\
\text { i }\end{array}$ & $\vec{\circ}$ & $\vec{\Xi}$ & & \\
\hline \multirow{3}{*}{ 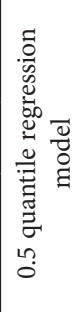 } & 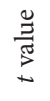 & $\begin{array}{l}\vec{b} \\
\stackrel{+}{+}\end{array}$ & $\begin{array}{c}0 \\
0 \\
1 \\
1\end{array}$ & 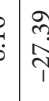 & 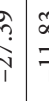 & 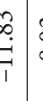 & $\begin{array}{c}0 \\
0 \\
0 \\
1 \\
1\end{array}$ & $\underset{T}{\stackrel{7}{i}}$ & $\begin{array}{l}\hat{0} \\
\dot{i} \\
\end{array}$ & $\exists$ & ชุ & $\hat{i}$ & $\stackrel{\leftrightarrow}{i}$ & $\underset{i}{\hat{i}}$ & $\underset{\hat{i}}{\hat{i}}$ & 苛 & $\overrightarrow{\tilde{o}}$ & 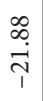 & $\stackrel{m}{m}$ & $\vec{i}$ & $\stackrel{\infty}{\rightarrow}$ & $\begin{array}{l}\overrightarrow{7} \\
\text { in }\end{array}$ & i & $\stackrel{0}{0}$ & 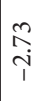 & $\underset{\sim}{\stackrel{f}{m}}$ & $\begin{array}{l}+ \\
\stackrel{0}{0} \\
1\end{array}$ & \\
\hline & \multirow{2}{*}{ 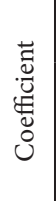 } & $\underset{x}{x}$ & 孛 & & $=\underset{\star}{x}$ & t & t) & 龸 & & & & 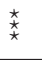 & $\begin{array}{l}\text { 孛 } \\
\star\end{array}$ & $\begin{array}{l}\text { 孛 } \\
\star\end{array}$ & $\begin{array}{l}\text { 杀 } \\
\star\end{array}$ & & & $\stackrel{\star \star ⿱ 乛}{\star}$ & $\underset{\star}{\star}$ & & $\underset{\star}{\star}$ & 坣 & & & 衤 & $\underset{\substack{x \\
x}}{*}$ & \multirow{2}{*}{\multicolumn{2}{|c|}{ 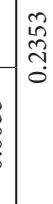 }} \\
\hline & & & 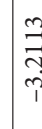 & 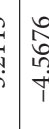 & 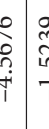 & 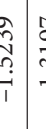 & $\begin{array}{l}\hat{\sigma} \\
\frac{\mathrm{m}}{i} \\
\end{array}$ & 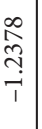 & $\begin{array}{c}\text { ô } \\
\text { co } \\
\text { i }\end{array}$ & $\begin{array}{l}\widetilde{\delta} \\
\stackrel{0}{\circ} \\
\stackrel{0}{0}\end{array}$ & 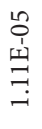 & 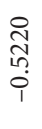 & $\mid \begin{array}{l}\hat{i} \\
\hat{i n} \\
0 \\
i \\
i\end{array}$ & $\begin{array}{l}\infty \\
0 \\
0 \\
0 \\
0 \\
1\end{array}$ & $\mid \begin{array}{c}\vec{\infty} \\
\infty \\
i \\
i\end{array}$ & 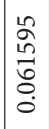 & $\mid \begin{array}{c}0 \\
\tilde{O} \\
\tilde{ָ} \\
0\end{array}$ & $\begin{array}{l}\text { 命 } \\
\hat{\imath} \\
i \\
i\end{array}$ & 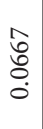 & $\mid \begin{array}{c}0 \\
0 \\
0 \\
0 \\
i \\
1\end{array}$ & \begin{tabular}{l}
$\infty$ \\
$\stackrel{2}{0}$ \\
\hdashline \\
$i$ \\
$i$
\end{tabular} & 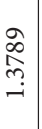 & $\begin{array}{l}\text { o̊ } \\
\text { ठे } \\
\text { i }\end{array}$ & 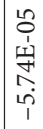 & $\begin{array}{l}0 \\
\overrightarrow{0} \\
\vdots \\
0 \\
i\end{array}$ & 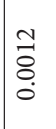 & $\begin{array}{l}n \\
\hat{o} \\
0 \\
i \\
i\end{array}$ & \\
\hline \multirow{3}{*}{ 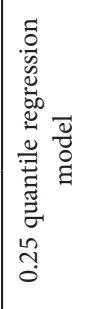 } & $\frac{\mathscr{\Xi}}{\tilde{E}}$ & فำ & 点 & 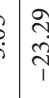 & 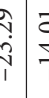 & 菅 & $\begin{array}{l}\vec{\sigma} \\
\text { ò } \\
i\end{array}$ & 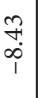 & $\begin{array}{l}\hat{6} \\
0 \\
0\end{array}$ & $\stackrel{\infty}{\stackrel{\sim}{\rightarrow}}$ & $\underset{\text { I }}{\stackrel{N}{.}}$ & ஒ̊ & 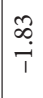 & त्t & $\overrightarrow{\hat{i}}$ & ふू. & $\mid \begin{array}{l}\mathscr{2} \\
i \\
i\end{array}$ & 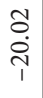 & $\stackrel{\sim}{\sim} \underset{+}{+}$ & in & 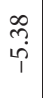 & $\stackrel{m}{m}$ & 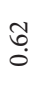 & $\underset{\stackrel{0}{0}}{i}$ & $\underset{\vec{i}}{\stackrel{\vartheta}{i}}$ & $\stackrel{\oplus}{=}$ & $\begin{array}{l}7 \\
7 \\
i \\
1\end{array}$ & \\
\hline & \multirow{2}{*}{ 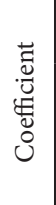 } & $\begin{array}{l}\substack{* \\
\star \\
\star} \\
\end{array}$ & $\stackrel{\star}{\star} \underset{\star}{\stackrel{\star}{*}}$ & $\stackrel{\star \star ⿱ 亠 乂}{\star}$ & 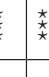 & 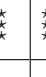 & 齐 & t. & & & * & $\underset{\star}{\star}$ & * & $\underset{\substack{x \\
x}}{*}$ & & & $\underset{x}{x}$ & $\underset{⿱}{\star}$ & $\underset{\star}{\star}$ & & 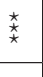 & $\underset{\star}{\star}$ & & & $\stackrel{x}{x}$ & & \multirow{2}{*}{\multicolumn{2}{|c|}{ 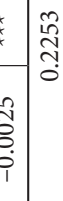 }} \\
\hline & & & 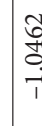 & 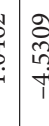 & 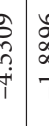 & 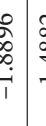 & 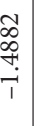 & 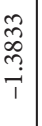 & $\begin{array}{l}\text { 苍 } \\
\stackrel{\Delta}{0} \\
0\end{array}$ & 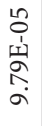 & $\begin{array}{l}\vec{\circ} \\
\stackrel{0}{\circ}\end{array}$ & $\begin{array}{l}m \\
\stackrel{m}{0} \\
\vdots \\
i \\
i\end{array}$ & 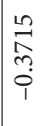 & $\begin{array}{l}\overrightarrow{0} \\
0 \\
0 \\
i \\
i\end{array}$ & $\begin{array}{c}\tilde{m} \\
\stackrel{n}{0} \\
i\end{array}$ & $\stackrel{\Re}{\stackrel{2}{二}}$ & $\mid \begin{array}{c}\infty \\
\stackrel{2}{7} \\
\stackrel{+}{i} \\
i\end{array}$ & 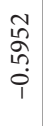 & $\begin{array}{l}\hat{\Omega} \\
\hat{\alpha} \\
0 \\
0 \\
0\end{array}$ & $\mid \begin{array}{c}F \\
\dot{0} \\
0 \\
i\end{array}$ & 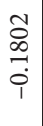 & $\begin{array}{l}\vec{\sigma} \\
\text { a }\end{array}$ & $\begin{array}{l}\stackrel{0}{0} \\
\stackrel{0}{0}\end{array}$ & 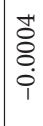 & $\begin{array}{l}\hat{o} \\
\text { ô } \\
\dot{i}\end{array}$ & $\begin{array}{l}\text { Lo } \\
\text { Oे } \\
0 \\
0\end{array}$ & & \\
\hline \multirow{3}{*}{ 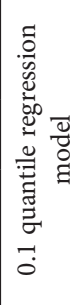 } & $\begin{array}{l}\frac{\mathscr{g}}{\tilde{E}} \\
\Xi \\
\Xi\end{array}$ & 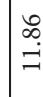 & $\begin{array}{l}\infty \\
0 \\
i\end{array}$ & 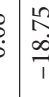 & 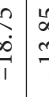 & : & के & 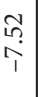 & $\stackrel{\Re}{\mathrm{i}}$ & $\begin{array}{l}\stackrel{\leftrightarrow}{\alpha} \\
i \\
i\end{array}$ & $\underset{\infty}{\infty}$ & $\begin{array}{l}\stackrel{m}{m} \\
\vec{i}\end{array}$ & $\begin{array}{l}\tilde{\rho} \\
\dot{i} \\
i\end{array}$ & $\underset{i}{\stackrel{+}{0}}$ & $\stackrel{\text { In }}{0}$ & $\stackrel{\sim}{\stackrel{\sim}{-}}$ & 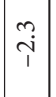 & $\begin{array}{l}\alpha \\
\hat{\sigma} \\
\hat{\tau}\end{array}$ & $\stackrel{\infty}{\stackrel{i}{\leftrightarrow}}$ & ĥे & 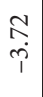 & $\stackrel{2}{-}$ & $\vec{m}$ & $\begin{array}{l}\overrightarrow{\vec{n}} \\
\overrightarrow{1}\end{array}$ & : & $\begin{array}{l}0 \\
0 \\
0\end{array}$ & {$\left[\begin{array}{c}\hat{n} \\
i \\
i \\
1\end{array}\right.$} & \multirow{3}{*}{ 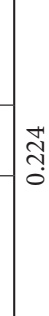 } \\
\hline & \multirow{2}{*}{ 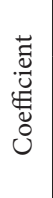 } & $\stackrel{x}{\star}$ & & $\underset{\star}{\star}$ & $=\underset{\substack{x \\
x}}{\underset{x}{x}}$ & t) & 齐 & 枽 & $\underset{⿱}{\star}$ & $\underset{\underset{x}{x}}{\stackrel{\star}{*}}$ & & $\underset{\star}{\star}$ & $\mid \underset{\substack{* \\
\star}}{ }$ & & & $\underset{t}{*}$ & $\underset{x}{\star}$ & $\underset{⿱}{\star}$ & $\underset{\star}{\star}$ & & $\underset{⿱}{\star}$ & * & & & * & & $\stackrel{\star}{\star}$ & \\
\hline & & 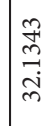 & 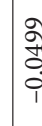 & & 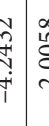 & 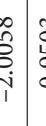 & 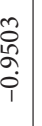 & 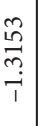 & 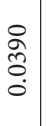 & $\begin{array}{l}\text { ồ } \\
\text { } \\
i \\
i\end{array}$ & 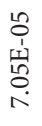 & $\begin{array}{l}\overrightarrow{\overrightarrow{0}} \\
0 \\
0 \\
i\end{array}$ & 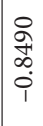 & 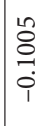 & $\begin{array}{l}\text { } \\
\tilde{z} \\
0 \\
0\end{array}$ & 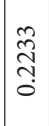 & 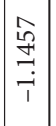 & $\begin{array}{l}\text { 苦 } \\
\text { hn } \\
0 \\
i \\
1\end{array}$ & $\begin{array}{l}\vec{F} \\
\stackrel{\overrightarrow{0}}{*}\end{array}$ & $\mid \begin{array}{l}\tilde{o} \\
\vdots \\
0\end{array}$ & $\begin{array}{l}\overrightarrow{I n} \\
+ \\
+1 \\
1\end{array}$ & 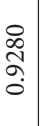 & $\begin{array}{l}\text { कि } \\
\text { के } \\
0 \\
0\end{array}$ & $\begin{array}{l}\infty \\
\text { oे } \\
0 \\
i\end{array}$ & $\begin{array}{l}\hat{0} \\
0 \\
0 \\
i\end{array}$ & $\begin{array}{l}0 \\
0 \\
0 \\
0 \\
i\end{array}$ & $\mid \begin{array}{l}\overrightarrow{0} \\
0 \\
i \\
\end{array}$ & \\
\hline \multirow{4}{*}{ 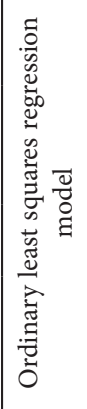 } & $\stackrel{|c|}{>}$ & & $\tilde{\Omega}$ & f & $f$ & هُ & نِّ & $\stackrel{\infty}{\stackrel{\infty}{+}}$ & 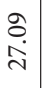 & $\begin{array}{l}\underset{\sigma}{0} \\
\stackrel{\infty}{\rightarrow}\end{array}$ & $\stackrel{F}{m}$ & $\stackrel{\widetilde{S}}{-}$ & $\underset{\rightarrow}{\stackrel{\Delta}{-}}$ & Î & $\underset{i}{\stackrel{2}{*}}$ & 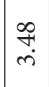 & $\underset{\substack{\infty \\
-i}}{\mid}$ & $\overrightarrow{\vec{i}}$ & $\begin{array}{l}\vec{\infty} \\
\dot{m}\end{array}$ & 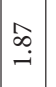 & $\stackrel{尺}{-}$ & 0 & $\stackrel{\widetilde{m}}{m}$ & $\begin{array}{l}\infty \\
\text { in } \\
6\end{array}$ & 命 & j. & $\mid \begin{array}{l}0 \\
\stackrel{0}{i} \\
i\end{array}$ & \\
\hline & 莺 & 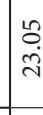 & $\stackrel{\overbrace{}}{\widehat{T}}$ & & î & 离 & 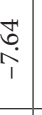 & 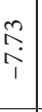 & 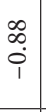 & $\stackrel{\sim}{\vec{r}}$ & $\stackrel{0}{\circ}$ & 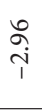 & 赵 & $\begin{array}{l}\infty \\
\infty \\
i \\
1\end{array}$ & $\underset{\sim}{\tilde{i}}$ & $\begin{array}{l}n ? \\
0 \\
0\end{array}$ & $\mid \begin{array}{c}\stackrel{L}{0} \\
\stackrel{i}{i}\end{array}$ & $\begin{array}{l}0 \\
\stackrel{1}{1} \\
\end{array}$ & î̉ & $\stackrel{\overbrace{}}{\ni}$ & ते & $\begin{array}{c}\vec{i} \\
i n \\
\end{array}$ & 3 & 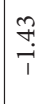 & 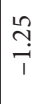 & 0 & $\mid \begin{array}{c}\infty \\
0 \\
\vdots \\
\vdots \\
1\end{array}$ & \\
\hline & \multirow{2}{*}{ 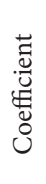 } & $\underset{x}{*}$ & t. & t. & 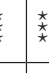 & & t. & 秝 & & $\underset{\star}{\star}$ & & $\underset{\star}{\star}$ & $\underset{\substack{\star \\
\star}}{*}$ & $\underset{x}{\star x}$ & $\stackrel{\star}{\star}$ & & 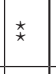 & 齐 & $\stackrel{\substack{x \\
x}}{x}$ & & * & 齐 & & & & & $\underset{\underset{x}{*}}{*}$ & \\
\hline & & 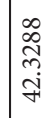 & $\begin{array}{l}\bar{D} \\
\tilde{a} \\
i \\
i\end{array}$ & & 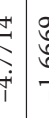 & . & 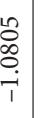 & 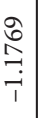 & 吕 & 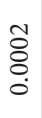 & 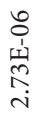 & 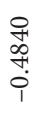 & $\begin{array}{l}0 \\
\text { in } \\
0 \\
i \\
1\end{array}$ & $\begin{array}{l}2 \\
2 \\
i \\
0 \\
i\end{array}$ & 㓂 & $\begin{array}{l}0 \\
\stackrel{2}{n} \\
\stackrel{0}{0} \\
0 \\
1\end{array}$ & $\mid \begin{array}{l}0 \\
0 \\
0 \\
0 \\
i \\
1\end{array}$ & $\begin{array}{l}\text { f } \\
\text { on } \\
0 \\
i\end{array}$ & $\begin{array}{l}\infty \\
\infty \\
0 \\
0 \\
0\end{array}$ & $\mid \begin{array}{l}\vec{\infty} \\
0 \\
0 \\
i \\
1\end{array}$ & $\begin{array}{l}\infty \\
\infty \\
0 \\
0 \\
i\end{array}$ & 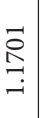 & & 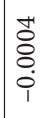 & 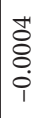 & o̊̀̊ & $\mid \begin{array}{l}\tilde{0} \\
0 \\
0 \\
i \\
0\end{array}$ & \\
\hline
\end{tabular}

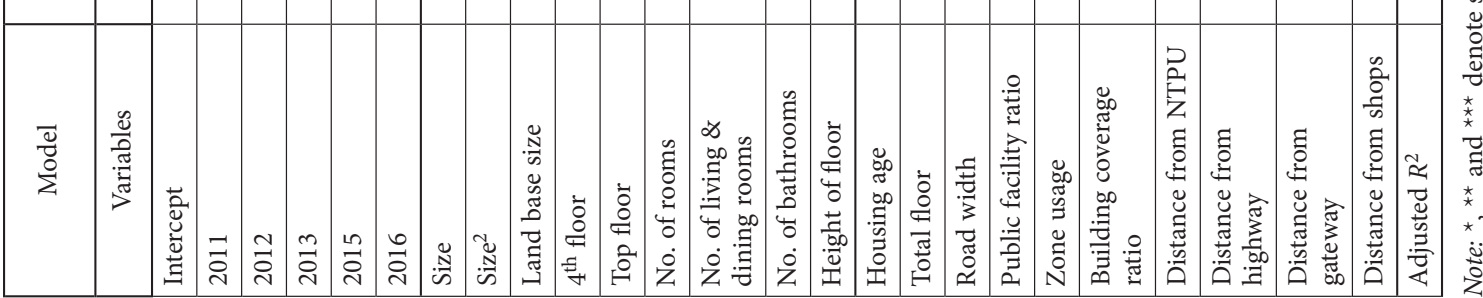


Table 6. Housing price-per-ping model - coefficient difference test results for different quantiles

\begin{tabular}{|c|c|c|c|c|c|c|c|c|c|c|}
\hline Model & $\begin{array}{r}0.1 \text { and } 0 \\
\text { quantil }\end{array}$ & & $\begin{array}{r}0.1 \text { and } \\
\text { quanti }\end{array}$ & & $\begin{array}{r}0.5 \text { anc } \\
\text { quant }\end{array}$ & & $\begin{array}{r}0.75 \text { an } \\
\text { quant }\end{array}$ & & 0.1 and 0.9 & uantiles \\
\hline Variables & Coefficient & $t$ & Coefficient & $t$ & Coefficient & $t$ & Coefficient & $t$ & Coefficient & $t$ \\
\hline 2011 & $4.2651^{* * *}$ & 4.38 & $0.9963^{*}$ & 2.75 & $3.1613^{* * *}$ & 5.82 & 1.1037 & 1.20 & -0.1220 & -0.20 \\
\hline 2012 & 0.4516 & 1.4 & 0.2876 & 1.36 & 0.3243 & 1.45 & 0.1272 & 0.48 & 0.2832 & 1.17 \\
\hline 2013 & $-0.4971^{\star \star}$ & -2.2 & -0.1162 & -1.53 & $-0.4819^{* * *}$ & -3.23 & -0.0151 & -0.08 & $0.3781^{\star *}$ & 2.09 \\
\hline 2015 & -0.2370 & -0.85 & $0.5379^{\star * *}$ & 3.46 & $0.3694^{*}$ & 2.46 & $-0.6064^{* *}$ & -2.09 & -0.0667 & -0.31 \\
\hline 2016 & -0.2497 & -1.31 & 0.0680 & 0.52 & -0.0775 & -0.35 & -0.1722 & -0.62 & 0.2927 & 1.09 \\
\hline Size & $0.1109^{\star * *}$ & 3.87 & $0.0316^{\star *}$ & 2.15 & 0.0410 & 2.10 & $0.0699^{*}$ & 2.48 & 0.0353 & 1.70 \\
\hline $\mathrm{Size}^{2}$ & $-0.0010^{\star * *}$ & -4.43 & $-0.0004^{\star * \star}$ & -3.31 & $-0.0005^{\star *}$ & -3.64 & -0.0005 & -2.53 & -0.0002 & -1.36 \\
\hline Land base size & $0.0002^{\star *}$ & 2.13 & $-4.20 \mathrm{E}-05$ & -0.47 & 5.93E-05 & 0.67 & $0.0002^{\star *}$ & 2.07 & $0.0001^{\star}$ & 1.93 \\
\hline $4^{\text {th }}$ floor & -0.0752 & -0.19 & -0.1997 & -0.94 & -0.0790 & -0.39 & 0.0039 & 0.01 & -0.0213 & -0.09 \\
\hline Top floor & $-1.3051^{\star * *}$ & -2.87 & $-0.4774^{*}$ & -1.60 & -0.3557 & -1.43 & $-0.9495^{\star * *}$ & -3.11 & $-0.4326^{* *}$ & -1.50 \\
\hline No. of rooms & $0.5164^{\star *}$ & 2.93 & $0.2159^{\star *}$ & 2.17 & $0.5703^{* * *}$ & 4.75 & -0.0539 & -0.22 & -0.1320 & -0.78 \\
\hline $\begin{array}{l}\text { No. of living and } \\
\text { dining rooms }\end{array}$ & 0.1945 & 0.89 & 0.1768 & 0.89 & $0.4255^{\star *}$ & 2.11 & -0.2310 & -1.16 & -0.2476 & -1.53 \\
\hline No. of bathrooms & $0.8527^{\star * \star}$ & 3.6 & 0.1059 & 0.77 & 0.1617 & 1.03 & $0.6912^{* * *}$ & 2.65 & $0.3631^{\star *}$ & 2.05 \\
\hline Height of floor & -2.0447 & -2.18 & 0.3261 & 0.43 & $-1.3485^{\star}$ & -1.70 & -0.6962 & -0.63 & -0.1792 & -0.25 \\
\hline Housing age & $0.2345^{\star \star \star}$ & 4.15 & 0.0388 & 1.06 & $0.1831^{\star * \star}$ & 4.06 & 0.0514 & 1.00 & -0.0525 & -1.04 \\
\hline Total floor & -0.0270 & -0.99 & 0.0188 & 0.95 & 0.0474 & 1.47 & $-0.0744^{\star * *}$ & -4.13 & -0.0068 & -0.24 \\
\hline Road width & 0.0158 & 0.86 & 0.0103 & 1.10 & 0.0144 & 1.62 & 0.0014 & 0.06 & 0.0030 & 0.17 \\
\hline Public facility ratio & 0.0596 & 1.64 & 0.0351 & 1.17 & 0.0332 & 0.68 & 0.0264 & 1.08 & 0.0334 & 1.38 \\
\hline Zone usage & -0.5877 & -1.2 & -0.0631 & -0.29 & -0.4509 & -1.54 & -0.1369 & -0.37 & 0.3813 & 1.11 \\
\hline Building coverage ratio & 0.0021 & 0.07 & 0.0335 & 1.92 & $0.0551^{\star}$ & 2.51 & $-0.0529^{\star * \star}$ & -3.06 & -0.0218 & -2.03 \\
\hline Distance from NTPU & 0.0008 & 1.04 & -0.0004 & -0.97 & -0.0007 & -1.47 & $0.0015^{\star *}$ & 3.24 & 0.0007 & 1.53 \\
\hline $\begin{array}{l}\text { Distance from } \\
\text { highway }\end{array}$ & -0.0004 & -0.49 & $6.77 \mathrm{E}-05$ & 0.16 & 0.0003 & 0.88 & -0.0007 & -1.06 & 0.0004 & 0.74 \\
\hline $\begin{array}{l}\text { Distance from } \\
\text { gateway }\end{array}$ & 0.0009 & 1.23 & $-0.0008^{*}$ & -2.48 & $-0.0015^{\star * *}$ & -3.47 & $0.0024^{\star * \star}$ & 4.99 & $0.0016^{\star \star}$ & 3.09 \\
\hline Distance from shops & 0.0009 & 1.21 & $0.0011^{\star *}$ & 2.58 & $0.0021^{* * *}$ & 4.54 & $-0.0012^{\star *}$ & -2.22 & -0.0004 & -0.74 \\
\hline
\end{tabular}

Note: ${ }^{*}{ }^{* *}$ and ${ }^{* * *}$ denote statistical significance at the $10 \%, 5 \%$, and $1 \%$ significance levels.

When comparing the results of Table 3 (total price model) and Table 5 (price-per-ping model), it is found that some of the variables (such as base size, number of living/dining rooms, the height of the floor, etc.) are different in terms of the coefficient values. This study believes that the main reason for this is that the purchasers in the housing market are highly heterogeneous, and the decision to purchase a home is quite complicated. When measuring the price-per-ping, high-priced housing usually reflects better locational conditions or construction quality. However, the purchaser may purchase a smallersized housing unit, so the result may not correspond to the high total price. When measuring the housing unit's total price, the affordability of housing is an important consideration or limitation of the purchaser. The size can thus be regarded as an important housing feature, and the size may exhibit a substitutionary relationship with other housing features.
As the height of the floor increases, the living space is less oppressive. The effect is also helpful to the improvement in housing quality. However, the construction cost of the developer will also increase, and so it will have a positive influence on the price-per-ping of housing. However, from the empirical results of the price-perping, only the 0.5 quantile has a positive influence on the price-per-ping, and it does not reach a significant level. At the 0.1 and 0.25 quantiles of the price-per-ping model, it even shows a significant negative influence, which indicates that the purchaser at the lower price-per-ping quantiles is less willing to replace other housing features with height of floor. Yet, as the height of floor increases, there is a positive influence on the total price. The increase in the number of living/dining rooms also helps to improve the quality of living. However, under a budget constraint, other living space may also be excluded. Based on the empirical results, it is found that the number of living/ 
dining rooms has no significant effect on the price-perping in the lower quantile range. However, at the 0.5 and 0.75 price-per-ping quantiles, there is a negative influence at the $5 \%$ significance level, which indicates that it there is a significant substitution effect on purchases of mediumand high-priced housing, and the overall influence on the price-per-ping is significantly negative. In terms of the total price model, the increase in the number of living/ dining rooms has a significant positive influence on the purchaser in between the 0.25 and 0.75 total price quantiles. There is a positive influence on the total price at the $10 \%$ significance level.

The influence of the land base size on the price-perping does not reach a significant level for most quantiles. Only at the 0.9 quantile does it have a negative influence at the $10 \%$ significance level. However, in the case of the total price model, although there is a positive influence at the 0.9 quantile at the $10 \%$ significance level, the overall influence is negative. Other than that, the price of the top floor in the OLS model has a significantly negative influence, and the total price of the OLS model has a negative influence but it is not significant. It is slightly different from the general cognition. This study believes that although the top floor offers a better view than other floors, it also raises the possibility of heat exposure from the sun or roof leakage. However, all building developments within the NTPU Special Zone are newly-developed buildings. In addition, the previous tradition of the top floor owner having the right to use the roof also does not exist in the Zone. As a result, the net influence of the top floor on the total price and the price-per-ping is negative most of the time, and only the price-per-ping at the 0.9 quantile exhibits a significantly positive influence.

Compared with the previous land-related studies, the assumption of larger-sized land having a quantity premium effect is supported by Tabuchi (1996), Colwell and Munneke (1999), and Thorsnes (2000). It considers that large-sized land can, relatively speaking, achieve largescale residential development, and the housing premium effect is the result of reflecting on the cost of the land premium effect. As for the inconsistency with the findings of Asabere and Colwell (1985), it may be the case that the research adopts the average size housing as the dividing point for the analysis. Moreover, the values of both ends are not considered. As for the smaller-sized housing, there is a discount effect on the total price. According to Colwell and Munneke (1999), the land price for most land sizes in the city exhibits a discount effect as the size increases, when the smaller parcels of land are developed into smaller-sized housing units. At the same time, the two can indeed reflect the cost of land. In other words, when designing a small-scale housing development, both land and housing have a quantity premium effect, which echoes the conclusions of Lin and Evans (2000).

When large-scale land is developed with average-sized housing in mind, although the land gives rise to a quantity discount effect, the house size may lead to a quantity premium effect. Clauretie and Li (2019) found that the price- per-ping of land increased initially as the size increased, and then decreased. However, after the land size exceeded 25 acres, the price-per-ping would be fixed. This is like the finding of this study in that the size in the price-per-ping model has a positive influence on the low quantile, and a negative influence on the high quantile, which is reflected by an interdependent response relationship. Clauretie and $\mathrm{Li}$ (2019) pointed out that the price-per-ping of land has a rigid characteristic, and this study points out that the total price of housing is similarly rigid.

\section{Conclusions and suggestions}

Previous studies have displayed strikingly different empirical results on the relationship between house size and price, and have led to many disputes. The empirical results of this study show that for house size that is either relatively large or small, the total price and price-per-ping will exhibit a nonlinear relationship with the increase in size. Based on the empirical study we draw some conclusions.

First, the total price of small-size housing will increase with the increase in size and the marginal total price will decrease. This is mainly due to the diminishing marginal utility of property rights. Such a concern has never been analyzed or discussed in previous studies. In comparison with the results of Veblen (1899) and Simlai (2014), the results of this study show that the application of the law of diminishing marginal utility to smaller size housing reveals the norm of a quantity discount effect. As the result does not coincide with the conclusion of a quantity premium effect as in Turnbull et al. (2006), it is probable that the research data is based on single-detached residential buildings. Such a type of building is distinctly different from the condominium-type buildings in the metropoli$\tan$ regions in Taiwan.

Secondly, the total price of large-sized housing will increase with the increase in house size and the marginal total price will also increase. This is obviously different from the traditional view that large-sized housing has a quantity discount effect due to the diminishing marginal utility. This quantity norm may be due to the conspicuous consumption characteristics, which confirms the quantity premium effect perception of Veblen (1899) and Lee and Mori (2016). However, it does not concur with the quantity discount perspective of Haurin (1988), Turnbull et al. (2006), Asabere and Huffman (2013), Simlai (2014) and other studies. As the property tax in Taiwan has no obvious differences in terms of the tax incentives related to house size, the empirical results of this study should be similar to those of Veblen (1899) but different from those of Hamilton (1976). The above empirical results can also logically explain that the developer will prefer to launch a housing project with large-sized housing to generate higher profits when the real estate market is booming. It also indicates that it is not reasonable for real estate appraisers to adjust the price-per-ping reduction for high-priced housing that is large. 
Thirdly, despite identifying the relationship between size and total price, the empirical results of this study also illustrate the importance of the relationship between size and price-per-ping. Through the traditional OLS model, the effect of size on price-per-ping is not significant. However, based on the QR, the size is found to have different influences on the housing price-per-pings that are relatively low and high. For housing with a relatively low price-per-ping, there is a relative price competitive advantage. The price-per-ping can increase as the size increases and the marginal price-per-ping decreases. Such a finding confirms the quantity discount effect perspective of Simlai (2014). Conversely, for housing with a relatively high price-per-ping, whether or not there is a conspicuous consumption characteristic, the price-per-ping will decrease as the house size increases and the marginal priceper-ping increases. Such a finding confirms the quantity premium effect perspective of Lee et al. (2006), Hui et al. (2016), and Li et al. (2015). As for the increase in the marginal price-per-ping, previous studies have not discussed the limitations of the price-per-ping. They ignore the rigidity of the equivalent total price curve pointed out in this study. In other words, because the price-per-ping in the high quantile area tends to reach the high total price band, an increase in the total price may easily exceed the burden or budget of the purchaser. As a result, the total price curves will no longer move outward, and the marginal price-per-ping will decrease.

This paper gives a good overview about the upcoming challenges and methods for house size and price modelling and can be regarded as a reference for others with its conceptual intention. Furthermore, our findings can provide very useful information for the product positioning decision of the developers, bargaining strategy of home purchasers, and as a reference of adjustment for real estate appraisers when conducting market comparison approach. If the governments have better knowledge of households' house size preference and their willingness to pay, then the planning of housing and urban renewal policy, the construction of public housing, valuation of the property tax base, and the compensation of eminent domain will also be better.

We have some suggestions for future research. First, although most previous studies confirmed that the house size has a nonlinear effect on the housing price, the turning point of the nonlinear relationship is different in different regions researched. This may be due to the different supply and demand structures of the real estate market in different regions. Secondly, to reduce the heterogeneity of neighborhood environment, this study has only adopted data samples from the NTPU Special Zone to be used in the empirical analysis. It is thus suggested that future studies could explore the relationship between house size and price in different regions. Thirdly, it is necessary to include more environmental characteristics and milieus of inhabitants as independent variables to build more sophisticated models. Furthermore, we measure the walking distance between the housing unit and its nearby facilities based on the OSM (open street map) which provided by the local government.
We would suggest to evaluate the use the Walk Score as a measure of neighborhood walkability. Finally, spatial geographic regression analysis could also be included to discuss the spatial dependence in different regions.

\section{Acknowledgments}

We thank the Ministry of Science and Technology, Taiwan for funding support (MOST104-2410-H-305-073-MY2).

\section{Conflict of interests}

The authors declare no conflict of interests.

\section{References}

Asabere, P. K., \& Colwell, P. F. (1985). The relative lot size hypothesis: an empirical note. Urban Studies, 22(4), 355-357. https://doi.org/10.1080/00420988520080591

Asabere, P. K., \& Huffman, F. E. (2013). The influence of relative size and home values. The Appraisal Journal, 3, 24-33.

Brownstone, D., \& de Vany, A. (1991). Zoning, returns to scale, and the value of undeveloped land. The Review of Economics and Statistics, 73(4), 699-704. https://doi.org/10.2307/2109409

Clauretie, T. M., \& Li, H. (2019). Land values: size matters. Journal of Real Estate Finance and Economics, 58, 80-110. https://doi.org/10.1007/s11146-017-9628-x

Colwell, P. F., \& Scheu, T. (1989). Optimal lot size and configuration. Journal of Urban Economics, 26, 90-109. https://doi.org/10.1016/0094-1190(89)90030-2

Colwell, P. F., \& Munneke, H. J. (1999). Land price and land assembly in the CBD. Journal of Real Estate Finance and Economics, 18(2), 163-180. https://doi.org/10.1023/A:1007714624700

Colwell, P. F., \& Sirmans, C. F. (1993). A comment on zoning, returns to scale, and the value of undeveloped land. Review of Economics and Statistics, 75(4), 783-786. https://doi.org/10.2307/2110041

Ecker, M. D., \& Isakson, H. R. (2005). A unified convex-concave model of urban land values. Regional Science and Urban Economics, 35(3), 265-277.

https://doi.org/10.1016/j.regsciurbeco.2004.04.003

Hamilton, B. W. (1976). Capitalization of intrajurisdictional differences in local tax price. American Economic Review, 66(5), 743-753.

Haurin, D. (1988). The duration of marketing time of residential housing. Real Estate Economics, 16(4), 396-410. https://doi.org/10.1111/1540-6229.00463

Hui, E. C., Zhong, J., \& Yu, K. (2016), Heterogeneity in spatial correlation and influential factors on property price of submarkets categorized by urban dwelling spaces. Journal of Urban Planning and Development, 142(1), 04014047. https://doi.org/10.1061/(ASCE)UP.1943-5444.0000270

Lee, K. O., \& Mori, M. (2016). Do conspicuous purchasers pay higher housing premium effects? Spatial and temporal variation in the United States. Real Estate Economics, 44(3), 726763. https://doi.org/10.1111/1540-6229.12115

Lee, K. O., \& Mori, M. (2019). Conspicuous consumption and household indebtedness. Real Estate Economics, 1-30. https://doi.org/10.1111/1540-6229.12305

Lee, H.-J., Chang, C.-O., \& Hua, C.-C. (2006). The relationship between floor area and unit price across different residential types in the Taipei metropolitan area. Taiwan Land Studies, 9(1), 63-87 (in Chinese with English abstract). 
Leguizamon, S. (2010). The influence of reference group house size on house price. Real Estate Economics, 38(3), 507-527. https://doi.org/10.1111/j.1540-6229.2010.00275.x

Li, L., Cheung, D., \& Sun, H. (2015). Does size matter? The dynamics of housing sizes and prices in Hong Kong. Journal of Housing and the Built Environment, 30(1), 109-124. https://doi.org/10.1007/s10901-014-9398-1

Lin, T., \& Evans, A. W. (2000). The relationship between the price of land and size of plot when plots are small. Land Economics, 76(3), 386-394. https://doi.org/10.2307/3147036

Ritter, M., Hüttel, S., Odening, M., \& Seifert, S. (2020). Revisiting the relationship between land price and parcel size in agriculture. Land Use Policy, 97, 104771. https://doi.org/10.1016/j.landusepol.2020.104771

Rosen, S. (1974). Hedonic price and implicit markets: product differentiation in pure competition. Journal of Political Economy, 82(1), 34-55. https://doi.org/10.1086/260169

Simlai, P. (2014). Estimation of variance of housing price using spatial conditional heteroskedasticity (SARCH) model with an application to Boston housing price data. Quarterly Review of Economics and Finance, 54(1), 17-30. https://doi.org/10.1016/j.qref.2013.07.001

Tabuchi, T. (1996). Quantity premia in real property markets. Land Economics, 72(2), 206-217. https://doi.org/10.2307/3146966
Thorsnes, P., \& McMillen, D. P. (1998). Land value and parcel size: a semiparametric analysis. Journal of Real Estate Finance and Economics, 17(3), 233-244.

https://doi.org/10.1023/A:1007772223239

Thorsnes, P. (2000). Internalizing neighborhood externalities: the effect of subdivision size and zoning on residential lot price. Journal of Urban Economics, 48(3), 397-418. https://doi.org/10.1006/juec.2000.2173

Turnbull, G. K., Dombrow, J., \& Sirmans, C. F. (2006). Big housing, little housing: relative size and value. Real Estate Economics, 34(3), 439-456. https://doi.org/10.1111/j.1540-6229.2006.00173.x

Veblen, T. (1899). Theory of the leisure class. MacMillan.

White, J. R. (1988). Large lot zoning and subdivision costs: a test. Journal of Urban Economics, 23, 370-384. https://doi.org/10.1016/0094-1190(88)90024-1

Zietz, J., Zietz, E. N., \& Sirmans, G. S. (2008). Determinants of house prices: a quantile regression approach. Journal of Real Estate Finance and Economics, 37(4), 317-333. https://doi.org/10.1007/s11146-007-9053-7

Zahirovic-Herbert, V., \& Chatterjee, S. (2011). What is the value of a name? Conspicuous consumption and housing price. Journal of Real Estate Research, 33(1), 105-125. https://doi.org/10.1080/10835547.2011.12091294 